\title{
La production de guano artificiel, une étape dans la professionnalisation des fabricants d'engrais : l'exemple d'Édouard Derrien à Nantes (1840-1860)
} "Artificial guano " a step in the professionalization of fertiliser manufacturers: The example of Edouard Derrien in Nantes (1840-1860)

\section{Philippe Martin}

\section{(2) OpenEdition \\ Journals}

\section{Édition électronique}

URL : http://journals.openedition.org/abpo/2979

DOI : $10.4000 / a b p o .2979$

ISBN : 978-2-7535-4130-6

ISSN : 2108-6443

\section{Éditeur}

Presses universitaires de Rennes

\section{Édition imprimée}

Date de publication : 15 avril 2015

Pagination : 161-190

ISBN : 978-2-7535-4128-3

ISSN : 0399-0826

Référence électronique

Philippe Martin, «La production de guano artificiel, une étape dans la professionnalisation des fabricants d'engrais : l'exemple d'Édouard Derrien à Nantes (1840-1860) », Annales de Bretagne et des Pays de l'Ouest [En ligne], 122-1 | 2015, mis en ligne le 15 avril 2017, consulté le 23 avril 2019. URL: http://journals.openedition.org/abpo/2979; DOI : 10.4000/abpo.2979 


\title{
La production de guano artificiel, une étape dans la professionnalisation des fabricants d'engrais : l'exemple d'Édouard Derrien à Nantes (1840-1860)
}

\author{
Philippe MARTIN \\ Doctorant en épistémologie, histoire des sciences et des techniques, \\ Centre François-Viète - Université de Nantes
}

Au début du XIX ${ }^{\mathrm{e}}$ siècle, le développement démographique et urbain en Europe nécessita l'accroissement de la production agricole par l'exploitation intensive des terres déjà cultivées et par le défrichement de nouvelles surfaces. L'insuffisance des stocks de fumiers de ferme pour la fertilisation de ces terres conduisit à la quête de nouveaux engrais, des engrais artificiels. Ces derniers, encore appelés engrais commerciaux, étaient pour les auteurs de l'époque, des " engrais qui ne se préparaient pas dans les fermes à l'aide des produits directs des exploitations rurales ${ }^{1}$ "; ainsi ils étaient définis par opposition aux fumiers de ferme considérés comme les engrais naturels par les cultivateurs. Parmi les engrais artificiels figurait le sous-ensemble des engrais industriels, des engrais manufacturés par des industriels.

Deux engrais artificiels dominèrent alors le commerce international : le noir résidu de raffinerie de sucre puis les guanos, et en particulier le guano du Pérou. L'un, dérivé du noir animal, était un rebut de l'industrie du sucre ${ }^{2}$, l'autre un produit trouvé dans la nature ${ }^{3}$. En ce qui concerne le noir résidu

1. BARRAL, Jean-Augustin, "Composition et fabrication des engrais commerciaux ", Journal d'Agriculture Pratique, $3^{\mathrm{e}}$ série, Tome II, janvier à juin 1851, p. 34-71.

2. Le noir animal ou charbon animal était constitué d'os carbonisés et broyés; il avait une propriété de décoloration. À ce noir animal étaient ajoutés du lait de chaux et du sang pour clarifier le sirop de sucre brut. Le résidu de l'opération de clarification était le noir résidu de raffinerie ou noir de raffinerie, constitué d'un mélange d'os carbonisé, de sucre, de sang et de chaux. Noir animal et noir résidu de raffinerie étaient des produits distincts, même si, par abus de langage, les auteurs parlaient de noir animal pour le noir résidu de raffinerie en précisant qu'il s'agit de noir pur ou noir vierge.

3. Substance organique formée par les excréments des oiseaux de mer, déposés depuis des siècles sur une multitude de points du littoral péruvien, et en particulier sur les îles 
de raffinerie, ses capacités de fertilisation des terres granitiques de l'Ouest de la France, constatées vers 1820, avaient déclenché un engouement chez les agriculteurs et fait de Nantes, entre 1830 et 1860 le premier port européen du commerce des noirs, qui débarquaient de toute l'Europe ${ }^{4}$. Quant au guano du Pérou, son point fort était d'être un engrais complet à forte teneur en azote, très efficace et facilement transportable dans les champs. Le premier chargement débarqua à Nantes en 1842 pour le négociant Harmange Jeune ${ }^{5}$. Mais ces fertilisants avaient des prix de plus en plus élevés et créaient une dépendance vis-à-vis du commerce international. De plus, le guano du Pérou présentait d'autres inconvénients : des livraisons irrégulières des négociants anglais ayant l'exclusivité de son commerce ${ }^{6}$; des réserves limitées à quelques dizaines d'années ${ }^{7}$. À la fois territoire de fabrique de " noir animal " du fait de ses nombreuses raffineries de sucre et important territoire du négoce des engrais artificiels, le port de Nantes vit se développer au milieu du XIX ${ }^{\mathrm{e}}$ siècle une industrie des engrais artificiels manufacturés destinés à se substituer en partie aux noirs et aux guanos.

Nous nous demanderons, dans un premier temps, quel fut le contexte scientifique et économique, qui amena l'industrie naissante des engrais à prospecter de nouvelles matières premières et nous essaierons de suivre l'émergence, dans les années 1830-1840, des engrais artificiels dans le port de Nantes dominé par le commerce des noirs résidus de raffinerie. Nous nous focaliserons ensuite sur le cas d'Édouard Derrien, fabricant nantais, en essayant de suivre la stratégie commerciale, relationnelle et industrielle, qu'il mit en œuvre pour amener à la maturité et légitimer, dans les années 1850, cette industrie des engrais artificiels avec un engrais composé, le guano artificiel à l'origine d'une nouvelle filière d'engrais. Nous terminerons en nous interrogeant sur les motivations et les procédés des industriels des années 1845-1855 qui, dans la lignée du guano artificiel de Derrien,

Chincha. Son exploitation pour le commerce international débuta en 1840, suite à l'indépendance du Pérou et à la stabilisation du régime politique péruvien.

4. En 1844, 168 navires arrivèrent dans le port de Nantes avec 221250 hl de noirs résidus de raffinerie et $25000 \mathrm{hl}$ étaient produits sur place, pour un coût total de 2700250 francs en employant 300 journaliers et 150 marins (BOBIERRE, Adolphe, Technologie des engrais de l'Ouest de la France, Nantes, L. Guéraud, 1848, p. 320-323).

5. Anonyme, Notice sur le guano, Nantes, Imprimerie W. Busseuil, 1845; PHELIPPEBEAULIEUX, " Rapport sur le guano, mis en comparaison avec les engrais Esmein et Gallard ", Annales de la société royale académique de Nantes et du département de la Loire-Inférieure, vol. 5 ( $2^{\mathrm{e}}$ série), 1844, p. 32-42. En 1845, 311 tonnes de guano furent importées dans le port de Nantes, puis les importations furent irrégulières avec notamment 1049 tonnes en 1848 et 463 tonnes en 1850 (Arch. dép. de Loire-Atlantique, 1ET H 261). En 1858, pour 45 navires important du guano des îles Chincha, $7 \%$ du tonnage étaient destinés au port de Nantes et $66 \%$ au port du Havre. En 1860, la France importa environ 40000 tonnes de guanos contre 300000 tonnes environ pour l'Angleterre (Arch. nat., F12/6859 et F 12/6860). Cf. MathiEw, W. M., "Peru and the British Guano Market, 1840-1870 ", The Economic History Review, New Series, vol. 23, no 1 (avril 1970), p. 112-128.

6. Un des principaux négociants britanniques était la maison londonienne Gibbs \& Son.

7. Mathiew, W. M., " Peru... ", op. cit., p. 112-128. 
proposèrent des engrais artificiels manufacturés, de qualité et performants, confirmant la marche vers la professionnalisation de ces fabricants.

En reconstituant la démarche industrielle de fabricants d'engrais artificiel de l'agglomération nantaise, cet article contribue à alimenter une historiographie pratiquement inexistante ${ }^{8}$ sur les acteurs de l'industrie des engrais dans l'estuaire de la Loire au XIx ${ }^{\mathrm{e}}$ siècle, avant l'avènement des engrais chimiques minéraux. Il aborde, d'une part, les enjeux de la réutilisation des résidus industriels et urbains par les fabriques d'engrais dépendantes de ressources étrangères coûteuses et limitées ${ }^{9}$, et d'autre part, les enjeux de définition et de qualité des engrais en liaison avec la réglementation du marché et la lutte contre la falsification ${ }^{10}$. Par ces deux aspects, il contribue à l'histoire des techniques : d'abord, par l'étude des initiatives d'entrepreneurs innovants confrontés à des risques de pénurie de ressources car, comme le rappela Denis Woronoff, « il n'est pas douteux que les changements [techniques] décisifs sont induits par la raréfaction d'une ressource ${ }^{11}$ "; ensuite, par son rattachement à une nouvelle approche de l'histoire des techniques qui, selon Liliane Hilaire-Perez, ne se limite plus aux " approches classiques [qui] valorisaient les évaluations quantitatives de la croissance et notamment les gains de productivité des équipements lourds " mais qui ouvre " la voie à la prise en compte de la qualité " et qui souligne " l'ampleur des innovations de produits et des améliorations de qualité ${ }^{12}$ ».

\section{Émergence des engrais artificiels}

\section{L'enjeu de la réutilisation pour la chimie agricole}

La quête de fertilisants est antérieure aux débuts du XIX ${ }^{\mathrm{e}}$ siècle ${ }^{13}$, mais à partir des années 1810, les recherches et expérimentations se succédèrent

8. Les principaux ouvrages d'histoire économique, agricole et industrielle évoquant ces questions sont les suivants : BouRRIGAUD, René, Le développement agricole au $19^{e}$ siècle en Loire-Atlantique, Nantes, Centre d'Histoire du Travail, 1994; FiéRAIN, Jacques, "Croissance et mutations de l'économie (1802-1914)", dans Bors, Paul (dir.), Histoire de Nantes, Toulouse, Privat, 1977; RochCongar, Yves, Capitaines d'industrie à Nantes au XIX $X^{e}$ siècle, Nantes, Édition MeMo, 2003.

9. BARLES, Sabine, L'invention des déchets urbains, France : 1790-1870, Seyssel, Champ Vallon, 2005.

10. JAS, Nathalie, Au carrefour de la chimie et de l'agriculture, Les sciences agronomiques en France et en Allemagne, 1840-1914, Paris, Éditions des archives contemporaines, 2001; BOURRIGAUd, René, Le développement agricole..., op. cit., p. 171-203.

11. Woronoff, Denis, "Conclusion " dans Coquery, Natacha, Hilaire-Perez, Liliane, Sallmann, Line et Verna, Catherine, Artisans, industrie, nouvelle révolution du Moyen Âge à nos jours, Lyon, ENS Éditions, 2004, p. 479-480.

12. HILAIRE-PEREZ, Liliane, " La négociation de la qualité dans les examens académiques d'invention au XvIII ${ }^{\mathrm{e}}$ siècle ", dans StanzIANI, Alessandro (dir.), La qualité des produits en France (XVIII -XIX siècles), Paris, Belin, 2003, p. 55-68.

13. Dès le Xvle siècle, Olivier de Serres recommandait d'enrichir le fumier, constitué des déjections des animaux, de déchets variés comme les cendres, les rebuts des cuisines, les balayures, les curages de fossés, la sciure de bois. Dans certaines terres, on savait 
afin d'exploiter au mieux les résidus des activités industrielles des villes. La crainte d'un appauvrissement de la terre par la ville, qui s'appropriait les matières fournies par les terres agricoles sans les restituer, s'accentua en effet dans l'esprit des chimistes et des agronomes des années $1820^{14}$. Cette volonté de réutilisation s'inscrivait aussi dans une volonté d'autarcie et d'autonomie par rapport au recours à des importations de ressources étrangères. Ange Guépin ${ }^{15}$ (1805-1873), médecin oculiste et chimiste nantais, élève d'Orfila, exposait cette évolution dans son cours public de chimie industrielle professé à Nantes en 1829 : «Mais combien d'industries [issues de la vente d'un troupeau de bétail] étaient inconnues de nos pères : il n'y a pas encore longtemps que, dans beaucoup de villes importantes, le sang inutile coulait sur le pavé, les rognures de peau et les autres débris de tanneries étaient jetés parmi les immondices; le noir animal ${ }^{16}$, aujourd'hui si recherché des agriculteurs, enfoui dans les fosses au sortir des raffineries comme cause d'infection; alors, la fabrication d'huile de pieds de bœuf, l'extraction du suif des os étaient inconnues; le sel ammoniac nous venait d'Égypte, le bleu de Prusse était à inventer ${ }^{17}$ ".

Les années 1840-1850 virent l'agriculture être investie par des chimistes et la chimie agricole se développer en Europe avec des personnalités comme Justus Von Liebig (1803-1873), professeur à Giessen en Allemagne, Anselme Payen (1795-1871), et Jean-Baptiste Boussingault (1801-1887), professeurs au Conservatoire national des arts et métiers à Paris. Selon Nathalie Jas, on commence à concevoir que l'agriculture puisse être définie, étudiée et analysée par l'intermédiaire des outils fournis par la chimie, c'est-à-dire l'analyse chimique et la mise en expérience ${ }^{18}$. Ces chimistes tentèrent d'expliquer le système de nutrition des végétaux et d'identifier les substances fertilisantes. Sans que soient évitées les controverses, telle

aussi apporter de la marne ou des calcaires tendres. Cf. BoulainE, Jean, Olivier de Serres et l'évolution de l'agriculture, Paris, L'Harmattan, 2002, p. 50-51.

14. Barles, Sabine, L'invention des déchets..., op. cit., p. 7.

15. Né à Pontivy, Ange Guépin était issu d'une famille de républicains éprouvés; son père, à la tête de la Fédération de l'Ouest, siégea comme député pendant les Cent-Jours. Il fit ses études au collège de Pontivy. Il se présenta à 19 ans à l'École polytechnique, mais en raison de l'appartenance de son père à la Fédération de l'Ouest et de son action révolutionnaire, son nom fut rayé de la liste des candidats. Il se tourna alors vers la médecine; en 1828, premier prix de chimie au concours de la faculté de Paris, il fut reçu docteur en médecine. Guépin vint s'installer à Nantes et épousa Adeline Lesant, fille d'un pharmacien nantais. En 1830, il fut nommé professeur à l'École de médecine. Entre 1830 et 1848 , Guépin déploya une activité prodigieuse, exerça la médecine, se consacrant plus particulièrement aux pauvres et aux déshérités, ne se faisant payer que par ceux qui en avaient les moyens. Il créa la première clinique ophtalmologique et donna ses cours à l'École de médecine (KAHN, Claude et LANDAIS, Jean, Nantes et les Nantais sous le Second Empire, Ouest Éditions \& Université Inter-Âges de Nantes, 1992, p. 282).

16. Il s'agit ici, non de "noir animal " au sens propre, mais de « noir résidu " de raffinerie.

17. GuÉPIN, Ange, " Cours gratuit et public de chimie industrielle à Nantes, discours prononcé par M. A. Guépin, Docteur-Médecin, à l'ouverture de ce cours le 19 novembre 1829 ", p. 17-18.

18. JAS, Nathalie, Au carrefour de la chimie..., op. cit., p. 44-53. 
la « querelle de l'azote " entre Liebig et Boussingault ${ }^{19}$, les rôles de l'azote et du phosphore et de leurs actions combinées furent mis peu à peu en évidence. Des analyses effectuées sur les résidus urbains, les immondices et les vidanges conduisirent aussi ces chimistes à montrer la nécessité de valoriser ces déchets et de reconstituer ainsi le cycle de la nature. Dans son ouvrage de 1840, Chimie appliquée à la physiologie végétale et à l'agriculture, Liebig mit l'accent sur ce cycle: "Tous les principes minéraux que l'on exporte avec les animaux, les grains ou les autres produits agricoles, peuvent être regagnés par les excréments liquides et solides, par les os et le sang des animaux tués; il ne dépend donc que de nous de rétablir l'équilibre dans la composition de nos terres en recueillant avec soin tous ces objets $^{20}$. " Adolphe Bobierre (1823-1881) ${ }^{21}$, chimiste à Nantes, s'inspirant des idées de Liebig, montra ainsi la contradiction consistant à importer des substances fertilisantes, telles que le noir résidu et le guano, pour fertiliser les terres de l'Ouest alors que des fertilisants, disponibles sur place à Nantes, étaient gaspillés : "On a, selon nous, trop méconnu dans l'Ouest de la France, où les résidus de raffinerie sont en si grande faveur, l'avantage immense qu'on retirerait de l'annexion de cet engrais aux détritus animaux, matières fécales, chairs musculaires, sang, etc. Comme nous l'avons dit, le phosphate de chaux, tel était le cri général des agriculteurs jusqu'à ce jour. Aussi, pendant qu'on faisait venir à grands frais les noirs de raffinerie de Russie, de Prusse, de Hambourg, de Paris, de Bordeaux, de Marseille, etc., par une bizarre et ruineuse contradiction, on établissait chaque jour à Nantes, dans une des villes importantes de la France, des conduits souterrains destinés à verser dans la Loire les déjections fertilisantes de près de cent mille individus ${ }^{22}$ !"

Ces idées pénétrèrent pleinement la société française et la limitation de la production des résidus inutiles, quels qu'ils furent, devint en effet un enjeu tout à la fois industriel, agricole, urbain, hygiénique, de plus en plus clairement affirmé ${ }^{23}$.

19. Controverse entre une assimilation de l'azote de l'air pour Liebig et dans le sol pour Boussingault (cf. JAS, Nathalie, Au carrefour de la chimie..., op. cit., p. 39).

20. LiEBIG, Justus, Chimie appliquée à la physiologie végétale et à l'agriculture, Paris, Librairie de Fortin, Masson et Cie, 1844 (2 édition), p 251.

21. Bobierre, surnommé le "Pierre l'Ermite des engrais ", joua un rôle important dans la réglementation du marché des engrais en Loire-Inférieure. Formé en chimie, il débuta comme préparateur du cours de chimie au laboratoire de la faculté de médecine de Paris, dirigé par Jean-Baptiste Dumas, académicien et co-fondateur de l'École Centrale. Après un passage dans des usines du Nord, il arriva à Nantes en 1846 pour l'usine chimique Cartier de la route de Rennes. Rapidement, il s'intéressa à l'agriculture et au commerce du noir résidu de raffinerie dans le port de Nantes. Dumas, ministre de l'agriculture et Gauja, préfet de Loire-Inférieure, appelèrent Bobierre à la réorganisation et à la direction du contrôle des engrais dans le département. À la tête d'un laboratoire, il devint alors, en 1850, vérificateur des engrais pour le département (ANDOUARD, Ambroise, Notice biographique sur Pierre-Adolphe BoBIERRE, Nantes, Imprimerie de $\mathrm{M}^{\text {me }} \mathrm{V}^{\mathrm{e}}$ Camille MELLINET, 1882; KAHN, Claude et LANDAIS, Jean, Nantes et les Nantais..., op. cit., p. 136-137).

22. Bobierre, Adolphe, Technologie des engrais..., op. cit., p. 208-209.

23. Barles, Sabine, L'invention des déchets..., op. cit., p. 121. 


\section{Émergence d'une industrie des engrais artificiels à Nantes}

Enclenché à la fin des années 1810, le développement de l'industrie du noir animal prit réellement son essor sous la Monarchie de Juillet, à un moment où les élites bourgeoises locales prenaient conscience de la nécessaire industrialisation de Nantes mais aussi en réponse aux besoins croissants des raffineries de sucre, qui se réorientaient vers l'océan Indien ${ }^{24}$. Á la même époque, en 1831, la création par le gouvernement d'un Conseil général de l'agriculture lança une véritable politique en faveur du secteur agricole; l'objectif était d'augmenter la production de blé pour la population, mais aussi pour l'exportation. L'extension des surfaces cultivables était l'un des facteurs essentiels de ce développement, et le Conseil général de Loire-Inférieure consacra des fonds considérables au défrichement des landes et à l'assèchement des zones marécageuses ${ }^{25}$. En 1832, la création des comices agricoles devait favoriser la diffusion des connaissances et des techniques agricoles modernes. En 1834, le département distribua aux comices de cantons 21000 francs sous forme de primes d'encouragement à l'agriculture.

C'est alors que les landes de l'Ouest, pour lesquelles l'écobuage et la jachère ne parvenaient pas jusque-là à améliorer le rendement, devinrent subitement fertiles grâce à l'usage d'un produit "miracle ", le noir résidu de raffinerie. Le noir animal, préparé pour la clarification des sirops de sucre, était utilisé deux ou trois fois dans la raffinerie puis jeté, devenant le noir résidu de raffinerie. La découverte dans les années 1819-1820 des propriétés fertilisantes de ces noirs résidus de raffinerie sur le sol argilo-siliceux des landes, dont il doublait les rendements moyens, est presque devenue un mythe fondateur, mythe dont les héros furent le négociant, futur maire de Nantes, Ferdinand Favre (1779-1867), et le chimiste et industriel parisien, Anselme Payen. L'origine des propriétés du noir résidu fut d'abord identifiée dans l'azote, mais peu à peu il s'avéra que l'auteur du miracle était le phosphate de chaux ${ }^{26}$ : le noir résidu renfermait 50 à $75 \%$ de phosphate de chaux et un peu d'azote organique. Toutefois son usage n'entra que peu à peu dans les mentalités, comme le relata Adolphe Bobierre, citant un article de L'Ami de la Charte, écrit en mai 1829 : "Un petit chargement de noir, arrivé de Bordeaux, fut mis en chantier où il resta invendu. Transporté plus tard à Pont-Rousseau, il fut lentement détaillé à bas prix à quelques cultivateurs de la Vendée, qui ne tardèrent pas à reconnaître la vertu active de ce nouvel engrais ". Les avis réitérés de la Société académique de Nantes, les expériences nombreuses faites aux environs de la ville, en Vendée et en Bretagne, ne tardèrent pas à le faire reconnaître. Bientôt, ce résidu, employé dans les environs de toutes les raffineries pour remblayer les terrains, fut

24. PÉtré-GrenoullLEAU, Olivier, L'argent de la traite : Milieu négrier, capitalisme et développement, un modèle, Paris, Aubier, 1998, p. 274.

25. BAGRIn, Sylvie et LAÉ, Frédéric, De la Loire-Inférieure à la Loire Atlantique, Histoire du Conseil Général, Nantes, Coiffard, 2011, p. 78-80.

26. BourRIGAUd, René, Le développement agricole..., op. cit., p. 154. 
déterré avec un empressement remarquable ${ }^{27}$. Des dépôts de noir de résidu des marchands et négociants envahirent alors la périphérie des villes de Nantes, Rezé et Chantenay.

Le potentiel du noir de raffinerie pour assurer le développement agricole de l'Ouest mit en branle le monde des négociants qui s'engouffrèrent dans ce créneau porteur. Les cultivateurs des départements de l'Ouest consacrèrent leurs fumiers aux céréales d'automne mais employèrent pour les ensemencements du printemps des engrais artificiels, notamment du noir de raffinerie, mais aussi des cendres, des charrées et de la poudrette ${ }^{28}$. Le commerce du noir de raffinerie, source d'enrichissement, voire de spéculation, devint très attractif. Sa valeur marchande augmenta considérablement puisqu'il valait seulement 2 francs l'hectolitre en 1820 et qu'en 1855, à son apogée, il se vendait 12 à 16 francs suivant la qualité et la provenance ${ }^{29}$. La présence dans le port de Nantes de cet important marché de noirs résidus et la forte demande de fertilisants encouragèrent le développement d'autres formes d'engrais artificiels non plus importées mais fabriquées localement.

Des industriels se positionnèrent sur ce marché en proposant des engrais artificiels manufacturés compétitifs en termes de prix grâce au réemploi de tous résidus industriels et urbains : la poudrette et les noirs animalisés, tels que les noirs composés ou les noirs factices. La poudrette était produite à partir de matières fécales séchées; les noirs composés étaient constitués de noir animal, de noirs résidus de raffinerie, de tourbe et de matières organiques animales (sang, matières fécales). Quant aux noirs factices, il s'agissait de noirs " composés de toute pièce et ne contenant que des substances animales organiques annexées aux terres calcaires ${ }^{30}$ ".

Ainsi, l'expérience d'Ange Guépin est significative de certains écueils rencontrés dans ces prémisses par l'industrie des engrais artificiels manufacturés. Guépin rassembla quelques associés et constitua une société en 1836, la société de La Divroëte, pour fabriquer un noir factice ${ }^{31}$. Guépin, saint-simonien, faisait alors partie des capacités nantaises, qui, ayant le sentiment du déclin du port de Nantes, avaient la volonté de le redresser

27. BOBIERRE, Adolphe, L'atmosphère, le sol, les engrais : leçons professées de 1850 à 1862 à la chaire municipale et à l'École préparatoire des sciences de Nantes, Paris, Librairie agricole, 1866, p. 238-240.

28. Arch. dép. de Loire-Atlantique, 7M 10, Rapport sur la situation agricole du département de Loire-Inférieure en 1847 (10 août 1847).

29. KaHn, Claude et LandaIs, Jean, Nantes et les Nantais..., op. cit., p. 135-136.

30. BERTIN, Georges-Hector, Statistiques des engrais du département de La Loire-Inférieure, Nantes, Imprimerie de Camille Mellinet, 1841, p. 2-6.

31. Frambourg, Guy, Un philanthrope et démocrate nantais - Le Docteur Guépin - 18051873 - Étude de l'action et de la pensée d'un homme de 1848, thèse pour le doctorat, faculté des Lettres de l'université de Rennes, 1964, f. 55-63; BouRRIGAUD, René, Le développement agricole..., op. cit., p. 135-142; Arch. dép. de Loire-Atlantique, 5M 373, Dossier MartinGuépin (1836); 5M 380, Dossier Le Comte de Goyon (1837-1838). 
avec de nouvelles industries ${ }^{32}$. De plus, son ami Devay, élève de l'institut agricole de Mathieu de Dombasle, fondé en 1826, lui avait communiqué son intérêt pour les questions agricoles. Comme l'expliqua l'homme de lettres et romancier, Ernest Ménard, un des actionnaires de cette société, leur objectif était de " produire un engrais utile par le mélange des matières animales liquides ou dissoutes dans un autoclave sous l'influence d'une chaleur prolongée. Acheter tous les chevaux bons à abattre, toutes les mauvaises viandes de bouchers et beaucoup d'immondices tels que le sang et les ventres des animaux tués, tant à l'abattoir que chez les fabricants de conserves alimentaires, y joindre encore les têtes et les intestins des sardines, ainsi que les animaux morts à la campagne de maladies contagieuses ou autres, pour obtenir du sang et d'autres solutions animales propres à former avec du charbon de tourbe un excellent engrais, voilà notre but ". Ménard précisait que cet usage des débris d'animaux morts ${ }^{33}$ permettait de " favoriser la salubrité de la ville en la débarrassant de beaucoup de matières putrescibles ", et insistait enfin sur l'innovation industrielle apportée : « Notre fabrication de noir est toute nouvelle à Nantes. C'est une industrie d'un grand avenir ${ }^{34}$. " La société disposait de deux sites de fabrication : l'un au Buzard de l'Abbaye à Chantenay-sur-Loire, près de Nantes, et l'autre aux Ponts-de-Cé, près d'Angers. L'imprimé publicitaire sur les " engrais de la Divroëte " proclamait, en mettant l'accent sur la compétence scientifique : "Ces noirs sont fabriqués d'après les procédés de M. Guépin, professeur de chimie, à Nantes, dans le but de rivaliser par leur énergie avec les bons engrais et surtout avec les meilleurs noirs résidus de raffinerie, sur lesquels à dose égale ils ont eu souvent le dessus ${ }^{35}$. " Des marchés furent passés avec des fournisseurs de tourbe, de sang d'abattoirs et de vidanges. Les associés, n'ayant aucune connaissance du marché des engrais, passèrent un contrat d'exclusivité avec les négociants nantais Despechers et Bonnefin pour la distribution de leur produit. Mais la qualité de ce noir factice ne suscita pas l'adhésion des acheteurs : son aspect était trop éloigné de celui du noir résidu de raffinerie et son efficacité fertilisante douteuse. En fait, selon René Bourrigaud, alors qu'il avait mis en avant ses qualités de chimiste, Guépin reprit l'analyse erronée du pharmacien nantais Hectot, qui pensait que les matières organiques azotées formaient l'élément actif du noir animal, et non le phosphate du noir d'os, et que par conséquent le noir de raffinerie pouvait être remplacé par de la tourbe calcinée ${ }^{36}$. Par ailleurs, les usines se heurtèrent à des problèmes d'organisation pour répondre aux

32. Le MAREc, Yannick, Le temps des capacités - Les diplômés nantais à la conquête du pouvoir dans la ville, Paris, Belin, 2000, p. 82.

33. L'usage par les cultivateurs des déchets d'animaux morts (sang, chairs) comme engrais avait été proposé dès 1830 par Anselme Payen. Cf. PAYEN, Anselme, Notice sur les moyens les plus simples d'utiliser les animaux morts, Paris, Imprimerie $\mathrm{M}^{\text {me }}$ Huzard, 1830.

34. Arch. dép. de Loire-Atlantique, 5M 373. Dossier Martin-Guépin (1836), courrier d'Ernest Ménard au préfet à Nantes le $1^{\mathrm{er}}$ septembre 1836.

35. FrambourG, Guy, Un philanthrope..., op. cit., p. 55-63.

36. Bourrigaud, René, Le développement agricole..., op. cit, p. 172-177; НЕстот, "Communication sur le noir animal, faite à la société académique par M. Hectot, phar- 
commandes passées. Finalement, la société de la Divroëte fit faillite en 1839, trois ans seulement après sa création, sous le coup des procès intentés par les acheteurs et les négociants revendeurs.

Ainsi dans ce début du XIX ${ }^{\mathrm{e}}$ siècle, à l'initiative de chimistes, de médecins, de négociants et de fabricants-négociants, émergea l'industrie des engrais dans l'agglomération nantaise, à Nantes même et dans ses villes périphériques comme Chantenay et Rezé. Elle était constituée d'ateliers de carbonisation d'os pour le noir animal, de dépôts de noirs résidus de raffineries, et de chantiers de mélanges de substances telles que des tourbes, des matières fécales, des matières organiques animales ${ }^{37}$. Toute leur activité gravitait autour du port et des flux maritimes d'importation essentiellement d'os, de noirs résidus de raffinerie et des flux fluviaux d'importation de tourbes de Montoir-de-Bretagne. La professionnalisation de tous ces industriels était encore incertaine entre négociants-spéculateurs, parfois habiles à la falsification par des mélanges de noir résidu avec un fort pourcentage de matières sans valeur fertilisante comme la tourbe, et fabricants-innovateurs proposant de nouveaux fertilisants. Les tentatives d'innovateurs comme Guépin se trouvèrent confrontées à une connaissance insuffisante des mécanismes de nutrition des végétaux, à un marché encore refermé sur une clientèle locale, à une qualité de produit non satisfaisante pour l'agriculteur, à une incapacité à industrialiser la production et à un marché fortement perturbé par la falsification.

\section{Le guano artificiel d'Édouard Derrien, un produit de qualité au service d'une légitimation}

Ce fut vers les années 1848-1850, période marquée par une crise agricole $^{38}$, qu'Édouard Derrien, négociant et fabricant de noir animal et d'engrais à Chantenay, s'empara du décret du préfet Gauja et engagea des études sur le guano artificiel. Dans cette deuxième moitié du XIX siècle, le besoin de fertilisants s'amplifiait suite à un nouvel élan des défrichements sous la double influence de la loi Favreau et de l'ouverture des chemins vicinaux de grande communication et d'intérêt commun.

macien (séance du 6 mai 1830) ", Annales de la société royale académique de Nantes et du département de la Loire-Inférieure, vol. 1, 1830, p. 272-277.

37. En 1843, le besoin des fabriques de Nantes pour produire 1000 tonnes de noir animal était estimé à 1500 tonnes d'os, soit 150000 francs. (BoBIERRE, Adolphe, Technologie des engrais..., op. cit., p. 101). Selon les statistiques de 1839-1847, la production de noir animal de Loire-Inférieure était en deuxième position nationale et représentait en valeur $18,7 \%$ contre $47,4 \%$ pour la Seine et $15,3 \%$ pour le Nord, (BARLES, Sabine, L'invention des déchets..., op. cit., p. 15).

38. Agulhon, Maurice, 1848 ou l'apprentissage de la république, 1848-1852, Paris, Éditions du Seuil, 1973, p. 102. Arch. dép. de Loire-Atlantique, 7M 10. Rapport sur la situation agricole du département de Loire-Inférieure en 1849 (15 août 1849) : "Les produits de la répurgation de la Ville de Nantes, ordinairement si recherchés ont, comme le Noir de Raffinerie, comme les engrais pulvérulents manufacturés, éprouvé une diminution considérable dans leur consommation". 
Édouard Derrien, né le 27 juin 1812 à Loctudy (Finistère), était inséré dans un réseau familial influent, lié au négoce nantais, à l'agriculture d'avantgarde et à l'État. Son père, Louis Derrien, était, au début de la Révolution française, entrepreneur de travaux de génie civil pour les fortifications militaires à Brest; son oncle maternel, Guillaume Bourgault-Ducoudray, négociant, fut président du tribunal de commerce de Nantes en 1819 et 1820 , président de la chambre de commerce de Nantes de 1823 à 1827 et siégea au Conseil Général du commerce jusqu'en 1834. Derrien était aussi lié à la famille de négociants De Tollemare par l'épouse de son oncle Guillaume : Jean-Jacques Charles De Tollemare, agent voyer en chef du département de Loire-Inférieure, fut témoin à son mariage. L'agronome Jules Rieffel, directeur de la ferme-école de Grand-Jouan créée en 1840 à Nozay, au nord de Nantes, était aussi un proche de sa famille par son épouse Henriette, fille de Guillaume Bourgault-Ducoudray. Adolphe Billaut était son cousin du côté de sa mère Marie-Anne Bourgault; cet avocat nantais fut député breton et il sera un homme de premier plan du Second Empire, ministre de l'Intérieur, puis ministre d'État de Napoléon III ${ }^{39}$. Son frère, William Derrien, en charge, en 1834, de la répurgation dans la ville de Nantes, était un homme à tout faire. Toutes sortes d'affaires semblaient bonnes pour lui : William fut entrepreneur de voitures publiques, fabricant de gélatine, inventeur d'un procédé de fabrication de " savon fossile ". Installé à la Moutonnerie à Nantes, il fabriqua de la poudrette à partir de 1838, puis déménagea à Chantenay à partir de 1840 pour fabriquer un " terreau animalisé ", appelé " poudrette calcaire ", mélange de matières fécales, de chaux vive et de cendres ${ }^{40}$. Édouard Derrien épousa, en 1846, sa nièce, Corine, fille de William Derrien.

C'est à l'Institut agricole de Mathieu de Dombasle à Roville-sur-Bayon en Lorraine, que Derrien fut formé. Première institution de ce genre en France, en 1826, elle dispensait un enseignement pratique pour les chefs de domaine ${ }^{41}$. Mathieu de Dombasle recommandait la lecture d'ouvrages d'agronomie, notamment ceux de l'agronome allemand, promoteur de la " théorie de l'humus " ${ }^{42}$, Albrecht Thaër, dont il était l'un des traducteurs en

39. Arch. dép. de Loire-Atlantique, 2Q 1547 ( $\left.{ }^{\circ} 54\right)$, 2Q 1554 ( $\left.{ }^{\circ} 77\right)$, 2Q $9602\left(\mathrm{n}^{\circ} 74\right)$, 2Q 9606 ( $\mathrm{n}^{\circ}$ 74). BouRrigaud, René, Le développement agricole..., op. cit., p. 255-256 et p. 276. PLESSIS, Alain, De la fête impériale au mur des fédérés. 1852-1871, Paris, Editions du Seuil, 1973, p. 44. Bovar, André, La Chambre de Commerce et d'Industrie de Nantes. 1700-1987, Saint-Herblain, Cid éditions, 1990 p. 47; [http://ollivier.yves1.free.fr/Etude\%20 d'une\%20descendance\%20\%20\%20-\%20DERRIEN.pdf].

40. Arch. dép. de Loire-Atlantique, 5M 377.

41. KNITTEL, Fabien, Mathieu de Dombasle, agronomie et innovation, 1750-1850, thèse de doctorat en histoire moderne sous la direction de Simone Mazauric et Jean-Pierre Jessenne, université de Nancy-II, 2007, p. 346, et « Mathieu de Dombasle (1777-1843) : un agronome acteur majeur de l'enseignement agricole en France " dans D'ENFERT, Renaud et FontenEAu, Virginie [dir], Espaces de l'enseignement scientifique et technique. Acteurs, savoirs, institution, XVII -XX ${ }^{e}$ siècles, Paris, Hermann, 2011, p. 45-49.

42. La " théorie de l'humus " a été développée par l'agronome allemand Albrecht Daniel Thaer. Elle faisait de l'humus du sol (matière organique du sol) la source principale de la matière sèche nécessaire à la nutrition des plantes (FELLER, Christian, " La matière orga- 
France. Dans son enseignement théorique étaient dispensés des cours de physiologie végétale, de géométrie, de botanique, d'arpentage, de culture forestière, des notions de physique et de chimie; mais en fait, il préférait l'enseignement par la pratique et prônait l'observation prudente des faits. Il organisait des observations dirigées dans le cadre des " conférences agricoles ", consistant en tournées agronomiques dans le domaine de Roville.

Les frères d'Édouard, Just Derrien, avocat, et William Derrien, étaient propriétaires d'un terrain dans la quartier de la Grenouillère à Chantenay en bord de Loire, dit Chantier Derrien, provenant des anciens chantiers navals des frères Crucy. C'est là, qu'Édouard Derrien installa sa fabrique de noir animal au début des années 1840. Il reprit, en 1851, l'usine de Rochery de noir animal mitoyenne de son terrain, elle-même présente depuis les années 1830. Avec l'arrivée du chemin de fer à Chantenay en 1851, son implantation industrielle était stratégique, disposant à la fois de la proximité des transports ferroviaires et maritimes pour ses approvisionnements et ses exportations. Il fera, ultérieurement, preuve d'innovation, dans cette activité du noir animal, en déposant des brevets pour l'amélioration du four à carbonisation d'os ${ }^{43}$.

Il existe de nombreux rapports issus des expositions nationales et internationales auxquelles Derrien participa, ainsi que des brochures commerciales, telles que Guanos artificiels spéciaux (1853), avec des témoignages, et Engrais Derrien, engrais artificiels complets et appropriés aux divers besoins de la culture (1862). Nous pouvons ainsi nous appuyer sur son cas pour suivre l'itinéraire commercial, relationnel et industriel d'un fabricant d'engrais de cette époque. Nous verrons, en effet, comment, bénéficiant d'un contexte scientifique et réglementaire favorable, sa formation agronomique, sa position dans les institutions, son expérience du négoce et sans doute ses talents d'entrepreneur permirent à Derrien de ne pas tomber dans les écueils du « noir factice " de Guépin, de faire reconnaître ces nouveaux engrais, de légitimer le métier de fabricant d'engrais et de développer une nouvelle filière d'engrais composés.

\section{Un nom " que tout le monde comprenne et prononce facilement"}

Convaincu de sa mission, Édouard Derrien estimait que le fabricant d'engrais avait un rôle central à jouer dans le développement agricole. Pour lui, la production de fumier était "bornée ", "lente et coûteuse ", aussi "l'industrie de la fabrication des engrais artificiels [était] un des besoins de l'époque " et même " une nécessité impérieuse de l'époque " et il prônait «la recherche d'engrais commerciaux d'une grande puissance et la création

nique des sols : aspects historiques et état des conceptions actuelles ", Comptes rendus de l'Académie d'agriculture de France, vol. 83, n 6, 1997, p. 85-98).

43. Arch. dép. de Loire-Atlantique, $9 \mathrm{M}$ 138, Registre des demandes de brevets d'invention, $n^{\circ} 274$. En 1863, " un système de four pour revivifier les noirs "; en 1866, " la fabrication et la mise en meule du sucre en tablettes dites ménagères ". 
d'engrais artificiels, autrement dit, dans ce derniers cas, l'utilisation et le retour à la terre, à l'aide de procédés industriels, de certains produits très actifs et d'un prix relativement réduit ${ }^{44}$ ".

Son nouvel engrais, Derrien l'appela " guano artificiel ". Il combinait des éléments nutritifs issus de mélanges de résidus : le sang sec, la chair desséchée, les débris de fabriques de conserves alimentaires, ceux de fabriques de colle, les débris de laine, des excréments de poulaillers, les os, les râpures de cornes, les coquilles de mer et les cendres de bois ${ }^{45}$. Il obtenait ainsi un titre de 5 à $7 \%$ d'azote et 20 à $40 \%$ de phosphate de chaux. Son engrais se positionnait sur le marché du guano du Pérou pour des agriculteurs désirant un engrais efficace, transportable, complet et concentré ${ }^{46}$. Lors du choix du nom de " guano artificiel ", Derrien avait connaissance du prix, que nous décrirons plus loin, offert par la Société Royale d'Agriculture d'Angleterre pour un substitut du guano. Ce nom devait marquer les esprits et mettre en confiance le consommateur. Derrien raconta comment il le conçut : "Je ne les avais pas appelés primitivement guanos artificiels : leur nom était " sels calcaires animalisés ", et ce titre les définit très bien. Mais, pendant deux ans qu'ils sont restés sous cette dénomination, pas un acheteur ne l'a employé; qu'y faire? Il faut bien un nom que tout le monde comprenne et prononce facilement. Celui de "guano artificiel " (mes engrais renferment bien la composition riche et multiple du bon guano naturel) est le premier que j'ai pu proposer, expliquant parfaitement et loyalement la nature de l'objet présenté. " Nous constatons, à travers sa première dénomination, la nette filiation de son engrais avec le "noir factice ", constitué, selon la définition vue précédemment, de "substances animales organiques annexées aux sels calcaires ", mais aussi l'héritage d'une terminologie de la " théorie de l'humus " avec des références à l'" animalisé " et au « calcaire " comme le faisait l'Abbé Rozier (1781-1805) ${ }^{47}$.

\section{Une démarche agronomique, rationnelle et expérimentale}

À la différence de Guépin, pour la mise au point de son " guano artificiel ", Derrien suivit une démarche rationnelle et expérimentale destinée, à confirmer la qualité de son produit : "Les principes théoriques sur

44. DERRIEN, Édouard, Guanos artificiels et spéciaux, Nantes, Imprimerie Charpentier, 1853 , p. 6-8.

45. BARRAL, Jean-Augustin, "Rapport fait par M. Barral... ", 1855, op. cit., p. 197-200.

46. DERRIEN, Édouard, Guanos artificiels..., op. cit., p. 18. Il est efficace avec $300 \mathrm{~kg}$ par hectare au lieu de $10000 \mathrm{~kg}$ avec du fumier

47. « La terre calcaire est donc la seule terre végétale, le véritable humus soluble dans l'eau et la seule qui établisse et constitue la charpente des plantes [...] si on amoncelle les plantes [...] si on les laisse se décomposer [...] on obtiendra, en dernière analyse, la terre calcaire pure, le véritable humus [...] Cultivateurs, ne songez qu'à créer ce précieux humus $[\ldots]$ qui est une vraie terre animalisée [...] la seule qui entre dans la composition des plantes ", cité par FELLER, Christian, "La matière organique des sols... ", op. cit., p. 85-98. 
lesquels est basée ma fabrication sont donc justifiés par la pratique ${ }^{48}$." Comme le précisèrent le jury de l'Exposition industrielle de Laval, en indiquant que "ses procédés scientifiques [étaient] basés sur les écrits de Thaër, Boussingault, Payen et Liebig ${ }^{49}$ ", Derrien s'appuya sur les travaux théoriques des chimistes agricoles. Toutefois nous n'avons pas beaucoup plus de détails sur ce recours à la théorie si ce n'est une proximité avec la "théorie de l'humus ", qui semble sous-jacente dans sa confrontation avec la " doctrine des engrais chimiques ${ }^{50}$ " de Georges Ville en 1868, que nous évoquons plus loin. L'échec d'un engrais, pour lequel Liebig avait déposé un brevet en Angleterre et en France en 1845, et pour lequel il avait fondé une manufacture près de Liverpool en association avec l'industriel James Muspratt, montra alors les limites de la production d'un engrais conçu selon des théories chimiques mais sans confrontation au terrain ${ }^{51}$. Jean Augustin Barral ${ }^{52}$ soutint par la suite que la fabrication d'un engrais n'était pas qu'une affaire de laboratoire : «Liebig, comme avait fait deux mois avant lui [...] un chimiste français distingué, Kuhlmann, pensait qu'il suffisait d'analyser les cendres des diverses plantes, et de répandre sur le sol précisément les quantités de sels minéraux que l'analyse indiquait comme devant être enlevées à ce sol pour les récoltes que l'on voulait obtenir. L'agriculture devenait une affaire de laboratoire et de fabrique de produits chimiques ${ }^{53}$."

Conformément à sa formation par Mathieu de Dombasle à Roville, Derrien appliqua les principes de son maître selon lesquels une théorie n'est rien si elle n'est pas vérifiée empiriquement ${ }^{54}$. Avant de commercialiser son produit, il l'étudia pendant trois ans dans une ferme expérimentale lui appartenant à Saint-Etienne-de-Montluc à proximité de Chantenay ${ }^{55}$. Il testa différentes proportions de ces substances selon la culture, son époque de végétation et sa composition chimique notamment, et il en vint ainsi à

48. Ibid., p. 46.

49. DERRIEN, Édouard, Guanos artificiels..., op. cit., p. 32.

50. LeCouteux, E., "La doctrine agricole de M. Ville ", Journal d'agriculture pratique, Tome premier, janvier à juin 1868, p. 129-137.

51. Site web INPI (brevets du XIX ${ }^{\mathrm{e}}$ siècle de l'Institut National de la Propriété Industrielle [http://bases-brevets19e.inpi.fr]) : Brevet cote 1BB1997 et intitulé " méthodes concernant la préparation d'engrais et leur application à l'agriculture ", enregistré en 1845 (BENSAUDEVINCENT, Bernadette et STEngers, Isabelle, Histoire de la chimie, Paris, La Découverte/Poche, 2001, p. 225).

52. Jean-Augustin Barral (1819-1884) fut nommé associé ordinaire en 1856 puis secrétaire perpétuel de la Société d'Agriculture de France en 1871 jusqu'en 1884. Élève de l'École polytechnique (1838), il entra dans l'administration des tabacs comme chimiste et découvrit la nicotine. Professeur de physique et chimie à Sainte-Barbe (1841-1852), répétiteur de chimie à Polytechnique (1845), il fut révoqué en 1849 . Arago le protégea et Bixio l'associa à la fondation du Journal d'agriculture pratique (BoulainE, Jean, Histoire de l'agronomie en France, Paris, Lavoisier Tec\&Doc, p. 287).

53. BARRAL, Jean-Augustin "Composition et fabrication... ", op. cit., p. 34-71.

54. KNITTEL, Fabien, Mathieu de Dombasle..., op. cit., p. 343-345.

55. DERRIEN, Édouard, Engrais Derrien, Engrais Derrien, Engrais artificiels complets et appropriés aux divers besoins de la culture, Nantes, Imprimerie Charpentier, 1862, p. 3. 
définir six classes d'engrais complets par une " classification rationnelle, basée sur l'étude de la physiologie végétale et sanctionnée par une pratique de douze années ${ }^{56}$ " comme il l'exprima dans les années 1860. Il conçut ainsi les " guanos artificiels " spéciaux numérotés avec le $\mathrm{n}^{\circ} 1$ pour le froment, le $n^{\circ} 2$ pour le blé noir, le $n^{\circ} 3$ pour le trèfle, le $n^{\circ} 4$ pour le choux et le $n^{\circ} 5$ pour les prairies artificielles. Derrien vantait son engrais comme "nouvel engrais rationnel " en précisant : " les principes théoriques sur lesquels est basée ma fabrication sont donc justifiés par la pratique ". Il redoutait de se " laisser entraîner par des idées théoriques loin de la vérité pratique ", pour lui, reprenant les principes de Mathieu de Dombasle, "l'agriculture est surtout une science d'observation ", et il voulait, selon son expression, " demander au sol et aux plantes leur opinion " et proclamait que " c'est au résultat général d'un ensemble de faits que l'expérience doit demander la vérité ${ }^{57}$ ". Selon Bobierre, le " guano artificiel » de Derrien était effectivement fondé "sur les déductions les plus positives de la science ${ }^{58}$ ".

\section{Une demande de réglementation du marché et l'arrêté préfectoral de 1850}

Un aspect majeur de la stratégie de communication commerciale de Derrien fut fondé sur la qualité de son produit, le "guano artificiel ", pour lequel il souhaitait que les agriculteurs puissent avoir toute confiance « à une époque où les engrais artificiels venaient de tomber si bas dans l'estime publique $^{59}$ " selon lui, faisant allusion aux falsifications de noirs et de noirs factices, auxquels son produit était apparenté.

L'obtention de la reconnaissance de la qualité des engrais fut le résultat des évolutions combinées des procédés d'analyse chimique liés aux progrès de la chimie agricole, de la réglementation du marché via la lutte contre la falsification et de la reconnaissance de l'" artificiel " vis-à-vis du « naturel ». Payen résuma toute cette problématique : «Malheureusement la fraude ne pouvait manquer de s'introduire dans ce commerce [des engrais à base de résidus des industries], comme elle s'est interposée déjà entre les raffineries et les consommateurs des résidus charbonneux de ces usines : on comprendra donc combien il est important de pouvoir déterminer d'une manière précise la richesse d'un engrais, puisque c'est le moyen sûr de moraliser ces sortes de transactions, d'introduire dans ce commerce l'habitude d'acheter et de vendre des marchandises suivant leur titre, d'activer le perfectionnement des engrais et d'accroître leurs débouchés en offrant aux consommateurs des garanties sur leur valeur réelle ou leur pureté ${ }^{60}$. " Et

56. Ibidem, p. 1.

57. DERRIEN, Édouard, Guanos artificiels..., op. cit., p. 11 et 45-46.

58. Anonyme, Exposition Universelle de Londres en 1862, Rapport du jury local sur les produits agricoles et industriels de la Loire-Inférieure admis à l'exposition, Nantes, V ve C. Mellinet, 1862.

59. DERRIEN, Édouard, Guanos artificiels..., op. cit., p. 24.

60. PAYEN, Anselme, Précis de chimie industrielle..., op. cit., p. 950. 
Barral de renchérir : "Le plus grand obstacle que rencontre la propagation des engrais artificiels, c'est la difficulté que l'on trouve à s'assurer de leur identité $^{61}$. " Dès 1830, Liebig proposa une méthode d'analyse élémentaire des substances organiques. En 1841, la méthode de Varrentrapp et Will permit de doser l'azote ${ }^{62}$. L'analyse chimique des engrais, par le dosage de l'azote et du phosphate, devint de plus en plus fiable, sans être encore parfaitement optimale, grâce aux procédés d'Eugène Peligot pour l'azote et de d'Augustino Malaguti pour le phosphate ${ }^{63}$. Ces avancées de l'analyse chimique étaient effectivement un prérequis à la lutte contre la falsification des engrais.

Cette lutte contre la falsification, de nature, de quantité et de qualité, fut précoce en Loire-Inférieure en raison de l'important commerce du noir résidu de raffinerie et de la fraude qui l'accompagna. Elle n'attendit pas la première loi répressive, qui ne vit le jour qu'en 1867, promue par des acteurs nantais comme Bobierre ${ }^{64}$. Face à un gouvernement d'idéologie libérale, l'administration préfectorale de Loire-Inférieure fut poussée à réagir par les agriculteurs, la Chambre de commerce, et des fabricants, comme Derrien, favorables à une réglementation du marché qui pût leur permettre d'exercer leur activité " loyalement " et de proposer des produits de qualité. Une succession d'arrêtés furent ainsi promulgués. Au " bureau d'essai des engrais ", à l'initiative du préfet Duval et du conseil général de LoireInférieure, en septembre 1837, succéda la première réglementation des engrais avec l'arrêté du préfet Chaper du 19 mai 1841 instaurant une déclaration pour tout nouvel engrais et son analyse, et enfin, les arrêtés du préfet Gauja des 23 février et 6 avril 1850 créant un « dépôt public " et une nouvelle organisation du contrôle fondée sur la composition chimique et accompagnée d'un étiquetage ${ }^{65}$. Ainsi, en 1850, le chimiste Adolphe Bobierre, fut nommé chimiste vérificateur en chef des engrais pour la Loire-Inférieure. Pour Nathalie Jas, avec cette réglementation préventive, ce sont désormais des normes qui vont définir ce qu'est un engrais. L'autorité préfectorale fait confiance à l'autorité de la science pour reconnaître le vrai du faux en matière d'engrais ${ }^{66}$.

Derrien décrivit l'impact favorable de l'arrêté préfectoral sur son activité : " Il est certain que jusque-là il était fort difficile de s'adonner à la fabrication d'engrais étudiés et loyaux; du moins, cela me semblait tel. Ainsi s'explique mon refus, depuis dix ans, de m'occuper de cette industrie [...].

61. BARRAL, Jean-Augustin, "Sur l'engrais Derrien ", Journal d'agriculture pratique, quatrième série, tome VII, janvier à juin 1857, p. 289-290.

62. Citation de W. Henneberg (1878) dans JAS, Nathalie, Au carrefour de la chimie..., op. cit., p. 51 .

63. PAyEn, Anselme, Précis de chimie industrielle..., op. cit., p. 927. MalaGuTi, Faustino, Leçons de chimie agricole, Paris, Dezobry, E. Magdeleine et Cie, Libraires-Éditeurs, 1855, p. $162-162$.

64. JAS, Nathalie, Au carrefour de la chimie..., op. cit., p. 64-66.

65. Bourrigaud, René, Le développement agricole..., op. cit., p. 171-203.

66. JAS, Nathalie, Au carrefour de la chimie..., op. cit., p. 61. 
Il ne suffit pas, en effet, de fabriquer de bons produits, il faut pouvoir les vendre; et lorsque le marché est tellement envahi par la fraude, qu'il faut user du même procédé pour obtenir une chance de succès, sans aucune apparence, au contraire, en faveur d'une marchandise loyale, le mieux n'est-il pas de s'abstenir ${ }^{67}$ ?"

\section{Des pratiques commerciales innovantes}

De cette réglementation, il sut tirer un avantage commercial, en étant, selon les auteurs de l'époque, le premier à proposer ses engrais avec leur analyse ${ }^{68}$, comme l'exposa Bobierre : "Les ventes de M. Derrien sont toutes faites sur analyses, qui consacrent une loyauté, d'ailleurs bien établie par de nombreuses transactions. [...] M. Derrien doit être félicité d'avoir le premier mis en pratique la vente sur analyses chimiques ${ }^{69}$. "Selon Barral, ces analyses étaient effectuées dans le laboratoire de Bobierre ${ }^{70}$. Derrien précisa lui-même les modalités de son engagement par rapport à ses analyses : "Pour garantie de la valeur de l'engrais que je livre, je remets entre les mains de l'acheteur un bulletin de vente portant l'analyse complète de l'engrais spécial livré. Les chiffres indiqués peuvent être contrôlés soit à la Préfecture, soit au Chantier Départemental, soit près du vérificateur officiel, soit enfin par tel chimiste expérimenté dans lequel l'acheteur a confiance $^{71}$. " Enfin Barral précisa que Derrien " garantit que son engrais a un dosage facturé; et tout acheteur aurait le droit d'obtenir ou la résiliation de son marché ou une indemnité si la composition du produit vendu n'était pas identique avec celle annoncée dans le contrat de vente ${ }^{72}$ ".

Derrien eut aussi recours à des témoignages d'agriculteurs " distingués " de toute la France, " au courant des nécessités de la science, sachant se servir de la balance comme base essentielle d'appréciation ", selon Barral ${ }^{73}$, et magnifiant les effets bénéfiques de son engrais.

67. DerRIEN, Édouard, Guanos artificiels..., op. cit., 1853, p. 9.

68. L'analyse, telle qu'elle était effectuée par le chimiste vérificateur en chef, fournissait les éléments suivants : pourcentage de charbon et matières organiques, silice, sels soluble, phosphates de chaux et carbonate de chaux; pourcentage d'azote (Arch. dép. de Loire-Atlantique, 7M 109, Rapport sur la situation des opérations faites au dépôt départemental d'engrais depuis le $1^{\text {er }}$ avril jusqu'au 30 inclusivement 1852).

69. Anonyme, Exposition universelle de 1855, Rapports de jury mixte international publiés sous la direction de S.A. Le Prince Napoléon, Président de la commission impériale, Paris, Imprimerie Impériale, 1856, p. 94-95.

70. Arch. dép. de Loire-Atlantique, 7M 109, «Extrait d'un rapport de MM. Barral \& Moll, délégués à Nantes par la Société d'Encouragement ", inclus dans le rapport de Bobierre sur la production et le commerce des engrais pendant l'exercice 1855-1856.

71. DERRIEN, Édouard, Guanos artificiels..., op. cit., p. 19.

72. BARRAL, Jean-Augustin, «Sur l'engrais Derrien », op. cit., p. 289-290.

73. BARRAL, Jean-Augustin, "Rapport fait par M. Barral, au nom du comité des arts chimiques, sur la fabrication des engrais artificiels de M. Édouard Derrien, ancien élève de Roville ", Bulletin de la Société d'Encouragement pour l'Industrie Nationale, tome 2, $54^{\mathrm{e}}$ année ( ${ }^{\mathrm{e}}$ série), 1855 , p. 197-200. 
Plus tard, en 1868, il lança la pratique des échantillons gratuits. Pour démontrer la performance de son engrais organique par rapport à celui de Georges Ville, il proposa d'organiser des études comparatives des engrais à partir d'échantillons gratuits. Il s'exprima ainsi dans le Journal d'agriculture pratique : "Je mets gratuitement à la disposition de messieurs les chefs d'école régionale d'agriculture, fermes-écoles, directeurs de domaines impériaux, présidents de comices ou cercles agricoles, fabricants de sucre et d'alcool, grands propriétaires ruraux et élèves des écoles régionales d'agriculture qui m'en feront la demande, et ce pendant trois années consécutives, chaque année, jusqu'à concurrence de 6000 kilogrammes de mes engrais spéciaux pour betterave ou froment ${ }^{74}$. "

\section{Une légitimation avec l'appui des réseaux savants}

Derrien s'appuya sur les réseaux de savants pour légitimer sa profession de fabricant d'engrais, s'intégrer dans l'élite du savoir et se distinguer de la concurrence. Il sollicita la caution de savants, qu'il obtint au travers de communications dans des bulletins de ces sociétés savantes et des institutions du monde industriel et agricole et via sa participation à des expositions avec remise de prix. Lui-même était membre de sociétés savantes, telles que la Société rovillienne ${ }^{75}$, la Société Académique de Nantes et de Loire-Inférieure et la Société d'Encouragement pour l'Industrie Nationale $(\text { SEIN })^{76}$ et fut un des correspondants pour la Loire-Inférieure, à partir de 1862, du Journal d'agriculture pratique fondé par Alexandre Bixio en 1837 et dirigé alors par Jean-Augustin Barral.

En premier lieu, il fit visiter son usine à une commission mixte du comité des arts chimiques et d'agriculture de la SEIN, constituée de Barral, professeur de chimie, et de Louis Moll ${ }^{77}$, professeur d'agriculture, et obtint ainsi

74. «Expérience sur les engrais ", Journal d'agriculture pratique, Tome premier, janvier à juin 1868, p. 532-533.

75. La société rovilienne était l'association des anciens élèves de l'Institut agricole de Mathieu de Dombasle à Roville-sur-Bayon.

76. Derrien fut élu résident de la Société Académique de Nantes et de la Loire-Inférieure le 5 février 1851 à la section Agriculture, puis au comité central de 1854 à 1856 (BLANLOEIL, Catherine, De l'institut départemental à la Société Académique de Nantes et de la LoireInférieure : une société savante de province au XIXe siècle (1798-1914), thèse de doctorat, université de Nantes, 1992, tome 3, p. 175). De plus, il fut membre en 1855 de la société Rovillienne (KnitTel, Fabien, Mathieu de Dombasle, agronomie et innovation..., op. cit., 2007,524 p.). Il fut aussi membre de la Société d'Encouragement pour l'Industrie Nationale (DERRIEN, Édouard, Guanos artificiels..., op. cit., p. 24).

77. Louis Moll (1809-1880) était originaire de l'Est de la France. Attiré par l'agriculture, il devint l'élève puis, à vingt ans, le collaborateur de Mathieu de Dombasle. En 1835, il publia un Traité de la science agricole car en 1831 il était devenu agriculteur pour son propre compte. En 1836, le Conservatoire des arts et métiers ouvrit un cours d'agriculture et une chaire d'agriculture fut créée en 1839. Il semble bien qu'elle fût la première chaire à avoir eu ce titre au monde et Louis Moll fut peut-être le premier à avoir porté le titre de professeur d'agriculture. En 1843, il fut nommé membre de la Société d'agriculture dont il sera président en 1865. Par ailleurs, il sera membre du conseil d'administration de la Société 
toute une série d'articles de Barral faisant la promotion de sa production de "guano artificiel " : en 1855, dans le Bulletin de la Société d'Encouragement pour l'Industrie Nationale (BSEIN), dans Le Progrès manufacturier, dans le Journal d'agriculture pratique; en 1857, de nouveau dans le BSEIN. Ce type de démarche, consistant, sur demande d'industriels, à rédiger des rapports d'expertise sur des procédés de fabrication, suite à des visites d'usines, pour encourager des productions nouvelles, était pratiqué dès les années 1825 à l'initiative de la Société académique de Nantes et publié dans le bulletin de la société Le Lycée Armoricain ${ }^{78}$, mais Derrien dépassa le cadre de visibilité local pour gagner un éclairage national. Il adressa aussi un courrier à l'Académie des Sciences pour qu'elle se prononce sur la valeur de son engrais à partir d'échantillons. Sa demande, " renvoyée à l'examen d'une commission composée de MM. Pelouze, de Gasparin et de Peligot ${ }^{79}$ " resta en suspens. D'autres articles furent encore publiés par des auteurs aussi reconnus que Bobierre, dans les Annales de la société académique de Nantes et de la Loire-Inférieure et dans les bulletins de diverses sociétés agricoles.

Enfin, il participa à de nombreuses expositions d'envergure nationale, avec l'Exposition universelle de 1855 de Paris, et internationale, avec celle de 1862 à Londres, reçut de nombreux prix au cours de la décennie 18521862 et insista sur la présence dans ces jury des chimistes et agronomes comme Adrien Gasparin (1798,1863), Anselme Payen, Amédée Boitel (18201884), Élie de Beaumont (1798-1874) ${ }^{80}$. Au-delà de la mise en valeur de son produit pour se démarquer de la concurrence, toutes ces démarches de Derrien visaient aussi à faire reconnaître le rôle du fabricant d'engrais et la qualité de son travail. C'est pourquoi il mit un point d'honneur à souligner que la remise, par la SEIN, de la grande Médaille de Platine en 1852 était « la seule récompense qu'elle ait jamais accordée à un fabricant d'engrais ".

\section{Le développement du marché et de la filière}

Une fois ses procédés mis au point, Derrien dut conquérir ce nouveau marché et s'occuper d'industrialiser sa production pour développer cette nouvelle filière du " guano artificiel " car, comme le rappelait Bobierre,

d'Encouragement pour l'Industrie Nationale en 1846 (BoulainE, Jean, « Louis Moll » dans FontAnon, Claudine et Grelon, André, Les professeurs du Conservatoire national des arts et métiers, dictionnaire biographique 1794-1955, L-Z, Paris, INRP/CNAM, 1994 , p. 273-276).

78. Le MARec, Yannick, Le temps des capacités..., op. cit., p. 84.

79. "Séance du lundi 19 juin 1854 », Compte rendu de l'Académie des Sciences, tome 38, janvier-juin 1854.

80. 1852, médaille de bronze au Concours Régional de l'Ouest; médaille d'or au Concours National à Versailles (Jury : Gasparin, Payen, Boitel, de Beaumont) et médaille d'or de rappel au Concours National suivant, Société d'Encouragement pour l'Industrie Nationale, grande Médaille de Platine.; 1859, Grande Médaille d'or Concours Régional de l'Ouest à Nantes; 1860, Médaille d'Or à l'Exposition Nationale Agricole de Paris; 1861, Diplôme d'Honneur à l'Exposition Nationale de Nantes; 1862, Participation à l'exposition universelle de Londres (DERRIEN, Édouard, Engrais Derrien, Engrais artificiels..., op. cit., 1862, p. 3-4). 
"Rien de plus facile que de fabriquer un échantillon d'engrais de quelques kilogrammes; rien de plus facile aussi que de donner à cet échantillon des propriétés excellentes et un prix fort minime. Mais à quoi bon de telles exhibitions, si en réalité la fabrication sérieuse et sur une échelle vraiment industrielle n'est pas un fait acquis ${ }^{81}$ ".

Au début des années 1850, les flux de fret de Nantes provenaient pour l'essentiel de son port, par navigation hauturière pour l'international et par cabotage pour le marché national car la Loire n'était pas suffisamment navigable pour acheminer toutes les marchandises. L'extension du chemin de fer et l'ouverture en 1851 d'une ligne directe de Nantes à Tours ouvrirent Nantes au marché national vers Paris via Tours d'un côté, et vers Bordeaux de l'autre ${ }^{82}$. Une gare fut construite au sud de Chantenay qui, prenant de plus en plus l'allure d'une zone industrielle, se trouva enserré entre une ligne de chemin de fer desservant le territoire national, le canal de Chantenay menant à la Loire et des estacades sur les rives de la Loire desservant les ports nationaux et internationaux avec des gabarres faisant des navettes jusqu'à Paimboeuf, l'avant-port de Nantes. L'usine de Derrien bénéficia de cette situation géographique pour ses approvisionnements et pour ses débouchés. En 1860, un tiers des importations nantaises de noirs résidus de raffinerie arrivait par voie de chemin de fer et les deux autres tiers par voie maritime ${ }^{83}$.

Pour sa fabrication de noir animal, Derrien recevait ses livraisons d'os par la Loire et les stockait dans des hangars construits au bord du canal de Chantenay. Le " guano artificiel " ouvrit de nouveaux courants d'échanges rendus possibles par le chemin de fer assurant le transport des matières premières et des chairs desséchées; le sang, les chairs et les débris d'os venaient de Paris et de Bordeaux ${ }^{84}$. Derrien profita de cette ouverture du marché national par le chemin de fer et de son expérience de négociant pour s'adresser aussi bien au particulier en direct qu'au commerce de détail et au commerce de gros en proposant des livraisons par wagons entiers. Dans une situation où une grande majorité d'agriculteurs était illettrée et se détournait des engrais industriels, le rail donna accès à Derrien à

81. BoBIERRE, Adolphe, «Rapport à la Société Académique sur l'exposition régionale de 1859 ", Annales de la société royale académique de Nantes et du département de la LoireInférieure, tome xxx, 1859, p. 609-610.

82. Jeulin, Paul, L'évolution du port de Nantes, organisation et trafic depuis les origines, Paris, Presses Universitaires de France, 1929, p. 331.

83. 2500 tonnes produits sur place, 8000 tonnes importés par chemin de fer, 16000 tonnes importés par voir maritime (DEHERAIN, Pierre Paul, Recherche sur l'emploi agricole des phosphates, Paris, Auguste Gouin éditeur, 1860, p. 17). Par exemple, les noirs résidus de raffinerie marseillais arrivaient par cabotage depuis Marseille jusqu'à Chantenay " aussi près de là qu'il pourrait aborder, prenant à sa charge les frais d'allège en Loire, s'il y en avait, et s'engageant dans ce cas à employer des gabarres pontées pour en faire la livraison " (Arch. dép. de Loire-Atlantique, 21U 63. Audience du tribunal de commerce de Nantes du 17 janvier 1863 entre Derrien et Codet).

84. Arch. dép. de Loire-Atlantique, 7M 10. Rapport au préfet de l'inspecteur d'agriculture, Jules Vidal, Nantes le 31 mars 1863. 
l'élite des " agriculteurs distingués, au courant des nécessités de la science [appartenant] aux départements de la Loire-Inférieure, du Morbihan, de Seine-et-Marne, des Deux-Sèvres, de Maine-et-Loire, du Pas-de-Calais, du Nord, etc. ${ }^{85}$ ".

En 1853, Édouard Derrien ajouta, dans son usine de noir animal, un atelier de revivification des noirs et un atelier de fabrication de " guano artificiel $^{86}$ ". Son commerce, tous engrais confondus, fut florissant : en 1854 furent vendues 400 tonnes d'engrais, en 1856, 1000 tonnes et en 1857, 2000 tonnes $^{87}$. En 1855, il produisit 400 à 600 tonnes de guanos artificiels ${ }^{88}$. Il accompagna la croissance du marché par des investissements de productivité grâce à l'ajout d'une machine à vapeur, de 15 chevaux-vapeurs puis de 18 chevaux-vapeur, et en augmentant les effectifs d'ouvriers de 50 à 80 entre 1855 et $1862^{89}$. Ces effectifs n'étaient pas négligeables car les statistiques industrielles de l'agglomération nantaise recensaient en 1856 6 fabriques d'engrais avec 70 ouvriers à Chantenay, et 10 fabriques avec au total une moyenne de 100 ouvriers selon la saison dans l'autre quartier industriel de l'agglomération, le quartier de la Prairie-au-Duc à Nantes, où en 1862, il existait 17 fabriques, avec un effectif de 168 à 269 ouvriers $^{90}$. En 1862, la production de noir animal de Derrien s'élevait à 9000 tonnes. Derrien exportait 200 à 700 tonnes de noir et d'engrais, notamment à La Réunion $^{91}$; il visait aussi les colonies anglaises, Madagascar et l'île de la Trinité dans les Antilles ${ }^{92}$. Bobierre put dire que "sa marque [était] estimée à Nantes comme aux colonies ${ }^{93}$ ". Comme il l'avait expliqué en 1859, Derrien réussit à passer au stade industriel et développer une nouvelle filière : "De nombreuses récompenses avaient déjà honoré la nouvelle branche de commerce [d'engrais industriel] implantée à Nantes par M. Derrien : la Chambre de Commerce avait hautement reconnu le ser-

85. BARRAL, Jean-Augustin, "Rapport fait par M. Barral... ", op. cit., 1855, p. 197-200.

86. 1844-1846, construction de sa maison; 1852-1853, construction d'une fabrique de noir (valeur 1700 francs); 1859-1860, construction d'une fabrique de noir (valeur 2400 francs); 1861, construction d'une fabrique de noir (valeur 1200 francs) (Arch. mun. de Nantes, $2 Z$ 487, commune de Chantenay, renseignements concernant les maisons et usines en construction ou reconstruction, 1842-1866).

87. BARRAL, Jean-Augustin, "Rapport fait par M. Barral, au nom du comité des arts chimiques, sur les engrais fabriqués par M. Derrien ", Bulletin de la Société d'Encouragement pour l'Industrie Nationale (BSEIN), tome 4, 56 ${ }^{\mathrm{e}}$ année ( $2^{\mathrm{e}}$ série), 1857, p. 146-148.

88. Anonyme, Exposition universelle de 1855, Rapports de jury mixte international publiés sous la direction de S.A. Le Prince Napoléon, Président de la commission impériale, Paris, Imprimerie Impériale, 1856, p. 94-95.

89. Anonyme, Exposition Universelle de Londres..., op. cit., 1862; BARRAL, Jean-Augustin, " Rapport fait par M. Barral... ", op. cit., 1855, p. 197-200. La première machine à vapeur date de 1842, d'après Daniel Pinson, L'indépendance confisquée d'une ville ouvrière, Chantenay, Nantes, ACL, 1982, p. 50.

90. Arch. dép. de Loire-Atlantique, 6M 900 et 6M 901. Statistiques industrielles.

91. Anonyme, Exposition Universelle de Londres..., op. cit., 1862.

92. Arch. nat., F12/6860. Lettre d'Édouard Derrien à Chantenay vers 1860.

93. Anonyme, Exposition Universelle de Londres..., op. cit., 1862. 
vice qu'il avait rendu sous ce rapport ${ }^{94}$. " Lors de l'Exposition nationale de Nantes en 1861, il reçut le diplôme d'honneur pour " fabrication et exportation importante d'engrais ${ }^{95}$ ". Son " guano artificiel " était vendu en 1854, 15 francs les $100 \mathrm{~kg}$ contre 30 à 35 francs pour le guano naturel, dont le prix s'élevait régulièrement en 1856 , avec toutefois un titre en azote de $5 \%$ contre $15 \%$ à $35 \%$ pour le guano naturel. Son prix monta à 20 francs les $100 \mathrm{~kg}$ en $1862^{96}$. Des critiques à propos de ses prix élevés au regard du taux d'azote se manifestèrent au fur et à mesure du développement de la concurrence $^{97}$.

Le " guano artificiel » fut un relais de croissance, il permit à Derrien de diversifier sa production, d'élargir sa gamme avec un produit innovant, qui avait une certaine valeur ajoutée sur son chiffre d'affaires. Fort de sa connaissance du marché du noir animal, il conquit ce nouveau marché en ayant une démarche de communication s'appuyant sur la caution savante et sur l'organisation des analyses, bien qu'encore imparfaites, dans le cadre de la réglementation sur la falsification des engrais de Loire-Inférieure, en avance sur la réglementation nationale. Derrien déploya ses compétences d'entrepreneur, comme les décrit François Caron ${ }^{98}$, en alliant dans la même personne, le scientifique, le fabricant et le négociant, c'est-à-dire le savoir, le savoir-faire technique et la connaissance du marché. Ainsi, il appliqua une démarche scientifique pour proposer un produit innovant, qui concourut à la reconnaissance du métier de fabricant d'engrais et qui initia, dans l'agglomération nantaise, une nouvelle filière technique réutilisant des rebuts industriels et urbains pour produire un " engrais artificiel».

\section{Les guanos artificiels dans les années 1845 à 1865 : vers la professionnalisation des fabricants}

La démarche d'Édouard Derrien ne fut pas isolée dans la période de 1845 à 1855, époque de l'ascension du guano du Pérou, elle s'inscrivit dans un engouement pour la réutilisation de matières premières locales réaffirmé par l'élite des scientifiques et stimulé par les Sociétés d'agriculture européennes. De nombreux industriels déposèrent alors des brevets pour des fertilisants organiques complets s'apparentant au guano artificiel. Certains reprirent même la terminologie, dont la paternité était revendiquée avec fierté par Édouard Derrien : «Les hommes de science et le public

94. BOBIERRE, Adolphe, « Rapport à la Société Académique sur l'exposition régionale de 1859 ", Annales de la société royale académique de Nantes et du département de la LoireInférieure, tome xxx, 1859, p. 609-610.

95. LefaIVRE, J., Les engrais à l'exposition nationale de Nantes 1861, Nantes, Imprimerie V. De Courmaceul, 1862.

96. DuDoüY, Alfred Léon, Guide pratique du cultivateur pour le choix, l'achat et l'emploi des matières fertilisantes, Paris, Librairie centrale d'agriculture et de jardinage, s.d., p. 164-167.

97. LefaIVRE, J., Les engrais à l'exposition nationale..., op. cit., 1862.

98. CARON, François, La dynamique de l'innovation, Changements techniques et changement social (XVI'-XXe siècles), Paris, Gallimard, 2010, p. 100-102. 
acheteur se sont donc entendus pour appeler un objet du même nom [le guano artificiel]; le fait est assez rare, en matière d'engrais surtout, pour être constaté ${ }^{99}$."

\section{La promotion par les Sociétés d'agriculture}

Dans le contexte, évoqué précédemment, de quête de nouvelles matières premières issues des rebuts industriels et urbains, les sociétés d'agriculture de France et d'Europe promurent le développement des engrais artificiels à partir de ressources locales. Dès les années 1845, la Société royale et centrale d'agriculture de France s'astreignit " à faire mieux comprendre les avantages de certains engrais riches indiqués dans [ses] concours et notamment des débris animaux si négligés autrefois et dont [ses] récompenses ont tant amélioré et répandu l'usage ${ }^{100}$ ". Face à l'augmentation constante du prix du guano du Pérou, dans les années 1850, les sociétés d'agriculture d'Angleterre, d'Ecosse et d'Irlande cherchèrent « à obtenir la création de fabriques d'engrais qui puissent remplacer le guano; elles ont fondé le prix pour l'invention de guanos artificiels, pour la découverte de nouveaux gisements d'engrais naturels, tels que les phosphates et azotates, pour le meilleur emploi de toutes les déjections et égouts des villes, etc. ${ }^{101}$ ". En 1852, la Société royale d'agriculture d'Angleterre offrit, en effet, un prix au fabricant d'engrais capable de livrer à un prix raisonnable un engrais équivalent au guano : "A Thousand Pounds and the Gold Medal of the Society will be given for the discovery of a Manure equal in fertilizing properties to the Peruvian Guano, and which an unlimited supply can furnished to the English Farmer at a rate not exceeding 5 l. per ton ${ }^{102}$."

\section{Une vague d'inventions de guanos artificiels}

C'est ainsi, qu'en France, plusieurs demandes de brevets d'invention de quinze ans furent déposées par des médecins, chimistes ou des manufacturiers entre 1845 et 1855 pour des engrais artificiels, de type guano artificiel : en 1845, le " guano factice " de la Société Louis Cherrier \& Cie à Paris; en 1847 , le "guano français " de Michel Édouard Esmein, médecin à Nantes; en 1851, le " guano de poisson " de Félix Demolon, frère de Charles Demolon, l'agriculteur et industriel de Concarneau, inventeur de l'engrais zoofime ${ }^{103}$;

99. DERRIEN, Édouard, Guanos artificiels..., op. cit., 1853, p. 13.

100. Arch. nat., F12/2407B. Anselme Payen, compte rendu des travaux de la Société royale et centrale d'agriculture du 14 avril 1844 au 30 mars 1845.

101. BARRAL, Jean-Augustin, "Rapport fait par M. Barral... ", op. cit., 1857, p. 146-148.

102. "Substitute for guano ", Journal of the Royal Agricultural Society of England, vol. 30, 1852. "Mais jusqu'à présent [en 1864] le prix n’a pas été mérité " (GIRARDIN, Jean, Des fumiers et autres engrais animaux, Paris, Victor Masson et fils, 1864 ( $6^{\mathrm{e}}$ édition), p. 41).

103. Charles Demolon déposa un brevet pour le "zoofime " en 1848. Son engrais était constitué de " soit [de] polypiers, maerl et madrépores, corps presque entièrement composés de carbonate de chaux [...] soit [de] phosphates de chaux si utiles aux plantes et [de] matières animalisées de toute sorte (chair musculaire, sang ou matières fécales 
en 1853, le "guano d'Europe " de De Villedeuil; en 1855, le " guano indigène " de Rohart, chimiste et industriel à Aubervilliers; en 1855, le " guano artificiel avec vidanges et matières fécales " d'Abendroth, docteur en philosophie et industriel de Dresde ${ }^{104}$. Jean Girardin ${ }^{105}$, professeur de chimie agricole, dans son ouvrage Des fumiers et autres engrais animaux, dans l'édition de 1876, en énuméra une liste plus exhaustive : " guano artificiel, guano urineux, guano indigène, guano Derrien, guano de Nantes, guano humifère, guano d'Aubervilliers, guano Fichtner, guano Abendroth, guano des Docks, guano de la Motte, guano agénais, guano de poisson, guano anglais, guano-phosphate, guano Millaud, guano animalisé, etc. ${ }^{106}$ ". Tous ces acteurs industriels étaient informés de leurs produits respectifs par leurs participations aux expositions industrielles et agricoles et par des revues comme le Journal d'agriculture pratique et, après 1860, par l'Annuaire des engrais et des amendements de François-Ferdinand Rohart.

La grande diversité de ces produits trouva son unité dans l'ambition de ces inventeurs de proposer un engrais complet, un substitut au guano du Pérou à un faible coût et composé de résidus industriels inutilisés et peu onéreux avec un fort taux d'azote : le sang des abattoirs pour la société Louis Cherrier \& Cie ; les matières fécales pour Esmein; la chair de poisson pour Demolon; l'eau ammoniacale des usines à gaz pour De Villedeuil; les résidus de fabriques de savon, de bougies et corps gras pour Rohart. C'est Abendroth, qui nous définit clairement l'enjeu industriel du " guano artificiel " en termes d'efficacité, de constance, de transportabilité, de manutention, de coût et d'industrialisation de sa production : " $1^{\circ}$ Que cet engrais puisse être fourni en quantité suffisante; $2^{\circ} \mathrm{Qu}$ 'il soit facilement transportable et que le maniement en soit facile et commode; $3^{\circ}$ Qu'il contienne les substances principales d'engrais dans des proportions toujours égales et que, $4^{\circ}$ La marchandise, présentant toutes ces conditions, puisse être établie à un prix modéré et en tout cas plus bas que le Guano du Pérou ${ }^{107}$."

Une des caractéristiques de la méthode des fabricants de ces engrais artificiels fut la mise à contribution de l'approche scientifique par des analyses chimiques du produit et par l'idée de synthèse. Rohart, chimiste,

préalablement désinfectées [...]) ». Cf. Site web INPI : cote 1BB7210. De l'engrais " zoofime " fut fabriqué par La Jarthe de Saint-Amand et $C^{\text {ie }}$ à Chantenay en 1851 (Étrennes nantaises, 1851).

104. Site web INPI : cotes 1BB2454 (Louis Cherrier), 1BB5081 (Michel Édouard Esmein), 1BB11069 (Félix Demolon), 1BB18379 (De Villedeuil), 1BB25556 (Rohart) et 1BB25599 (Abendroth).

105. Jean Girardin (1803-1884), élève de Louis Jacques Thénard, fut d'abord titulaire de la chaire municipale de chimie à Rouen (1828-1858), puis professeur et directeur de l'École préparatoire à l'enseignement supérieur des sciences et des lettres de la ville (1855-1858), puis recteur de l'académie de Clermont-Ferrand (1868-1873) (BIDOIS, Anne et SouLARD, François, "Entre sciences et industrie chimique : la carrière provinciale de Jean-Pierre Louis Girardin (1803-1884), savant, enseignant et vulgarisateur " dans D'ENFERT, Renaud et FontenEaU, Virginie [dir], Espaces de l'enseignement scientifique..., op. cit., p. 119-129). 106. GiRARDin, Jean, Des fumiers..., op. cit., 1876 (7 édition), p. 57-58.

107. Site web INPI, cote 1BB25599. 
effectua une analyse chimique sur les résidus de corps gras des abattoirs " après les avoir étudiés dans leur constitution et dans leur composition intime " pour y identifier " les mêmes éléments [que le guano naturel] et une grande quantité d'autres ${ }^{108}$ ". Louis Cherrier souhaita obtenir une synthèse pour la composition de son "guano factice" qu'il exprima ainsi : " Ayant recours à la science, nous lui avons demandé si parmi les résidus employés jusqu'ici [os, urine humaine, sang], il ne serait pas possible de confectionner par la synthèse un Guano factice ${ }^{109}$. "Par ailleurs, nous savons que la Société Barouet et Cie de Louis Cherrier avait recours à l'analyse d'échantillons d'autres engrais qu'elle produisait pour en garantir la qualité et la constance sur les différentes parties de territoire où elle était installée ${ }^{110}$. Ainsi, la pratique de ces fabricants de guano artificiel ciblant la qualité des produits fabriqués concourut à la reconnaissance de leurs produits et traduisit leur professionnalisation.

\section{Innovation par agencement nouveau de procédés disponibles}

Les brevets de ces inventeurs aboutirent à de réelles innovations de produits avec des engrais fabriqués industriellement et mis sur le marché. Nous pouvons leur ajouter les réalisations des fabricants de guano artificiel de l'agglomération nantaise à la suite de Derrien : le pharmacien Édouard Moride vers $1857^{111}$; Benjamin Leroux, fabricant de noir animal dans le quartier de la Prairie au Duc à Nantes vers 1858, après avoir déposé des brevets pour un " noir calcaire d'engrais " dans les années 1853-1854"112; Gustave Mongin $^{113}$, chimiste, fabricant à Nantes route de Rennes puis à Chantenay au Buzard de l'Abbaye vers 1858.

108. Site web INPI, cote 1BB25556.

109. Site web INPI, cote 1BB2454.

110. La fabrique de Duboy, directeur de la compagnie des engrais nantais au Bignon, représentant de la société Barouet et Cie, produisait un engrais constitué d'un mélange de matières fécales à demi liquides, avec de la terre calcinée, carbonisée ou seulement desséchée. Le siège de cette société à Paris imposait à ses fabriques le contrôle de la qualité d'un échantillon de leur production avant d'autoriser leur livraison au commerce (Arch. dép. de Loire-Atlantique, 5M 52).

111. Arch. dép. de Loire-Atlantique, 21U 59. Audience du tribunal de commerce de Nantes du 11 mai 1861 entre Cormillier et Chauveau. Édouard Moride est co-auteur avec Adolphe Bobierre des Études chimiques sur les eaux de la Loire-Inférieure, considérés au point de vue de l'hygiène et de l'industrie, en 1846, et de Technologie des engrais de l'ouest de la France, en 1848.

112. Benjamin Leroux installa une usine de charbon artificiel et torréfaction d'os à la Prairie-au-Duc à Nantes en 1863 (Arch. dép. de Loire-Atlantique, 5M 318). Dépôts de brevets de quinze ans, en 1853 pour " l'emploi de cornues à la fabrication de carbonate d'engrais " avec une addition en 1854 pour la " fabrication d'un noir calcaire d'engrais " (Arch. dép. de Loire-Atlantique, 9M 136-137, Registre des demandes de brevets d'invention, $\left.\mathrm{n}^{\circ} 185, \mathrm{n}^{\circ} 235\right)$.

113. Gustave Mongin déposa un brevet en 1863 pour " un four à carboniser dit de G. Mongin " (Arch. dép. de Loire-Atlantique, 9M 138). 
Comme l'exprima Rohart, la plupart des procédés de fabrication de guano artificiel n'étaient pas en eux-mêmes innovants car ils faisaient partie d'un savoir-faire partagé, mais ils étaient innovants en tant qu'agencement nouveau de techniques disponibles : "Ce ne sont donc point des procédés de fabrication que je viens faire breveter car les procédés que j'emploie peuvent varier à l'infini, et sont tous dans le domaine public. Je n'entends donc faire breveter ici que l'idée de la transformation de ces matières en guano ${ }^{114}$. "Néanmoins, certains procédés furent adaptés au traitement des constituants du guano artificiel et furent à l'origine d'innovations de procédés : des techniques de dessiccation et de désinfection pour en faciliter la manutention, le transport à distance et éviter la putréfaction et les odeurs. La société Louis Cherrier \& Cie proposa un procédé pour dessécher le sang; de Molon et Benjamin Leroux des procédés pour dessécher les chairs; la société Louis Cherrier \& Cie, Esmein, et Abendroth, des procédés de désinfection des matières fécales. Esmein proposa l'usage de la suie et du " proto-sulfate de fer " pour désinfecter les matières fécales. Benjamin Leroux, qui utilisait des déchets de cornes, de laine et des os, pour composer son guano artificiel eut "l'idée de les torréfier à 300 degrés pour les désagréger, sans causer une notable déperdition de leurs principes volatils [...] dans le but d'accélérer l'assimilation de leurs principes azotés ${ }^{115}$ ". Leroux déposa un brevet pour son procédé : en 1858 pour " la dessiccation et la torréfaction de matières contenants de l'azote propre à l'agriculture et d'une décomposition difficile tout en lui conservant la plus grande portée fertilisante", avec une addition en $1860^{116}$.

Ces manufacturiers mirent en œuvre des procédés avec des techniques artisanales issues des siècles précédents ${ }^{117}$ : carbonisation des os pour les noirs animal, distillation des gaz, fermentation pour les noirs résidus de raffinerie et les matières fécales, mélanges à sec, mélanges humides, mélanges activés par la chaleur. Ils esquissèrent néanmoins l'usage de produits chimiques, soit pour la dissolution, soit pour la désinfection. Ainsi Derrien traitait les os de qualité inférieure, rebuts de sa fabrication de noir animal, avec de l'acide sulfurique selon des procédés anglais du superphosphate de chaux ${ }^{118}$. Il n'opérait pas lui-même la dessiccation des chairs, il se les procurait toutes prêtes. Quant à Mongin, dont le guano artificiel était

114. Site web INPI, cote 1BB25556.

115. Anonyme, Exposition Universelle de Londres..., op. cit., 1862.

116. Arch. dép. de Loire-Atlantique, 9M 137. Registre des demandes de brevets d'invention, $\mathrm{n}^{\circ} 67, \mathrm{n}^{\circ} 168$.

117. Il s'agit souvent de procédés issus des techniques alchimiques sans les aspects ésotériques (cf. BAUDET, Jean, De l'outil à la machine, histoire des techniques jusqu'en 1800,

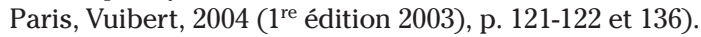

118. Le procédé de fabrication du superphosphate breveté par le manufacturier agronome John Bennet Lawes en 1843 et repris par James Muspratt fut appliqué en Angleterre aux os puis aux phosphates minéraux (HABER, L. F., The chemical industry during the nineteenth century, a study of the economic aspect of applied chemistry in Europe and North America, London, Oxford University Press, 1858, p. 59-62). Des controverses scientifiques en limitèrent l'usage aux os en France (Boulaine, Jean, La bataille des phosphates au 
constitué d'un mélange de noirs purs, de cendres d'os et de tourteaux de débris d'animaux, il se procurait, à Paris, ses tourteaux préparés par un traitement à l'acide sulfurique et par une soumission à la pression de fortes presses hydrauliques ${ }^{119}$. Nous voyons ici poindre une industrie des engrais réalisant des transformations des matières premières en ayant recours aux produits de la " grande industrie chimique".

\section{La fin du guano artificiel}

Si des industriels de l'agglomération nantaise, comme Gustave Mongin ${ }^{120}$ et Benjamin Leroux, suivirent l'exemple de Derrien et développèrent cette nouvelle filière, d'autres fabricants négociants de noir animal, bien implantés à Chantenay et renommés, comme la société Pilon père, Perthuy \& Cie présente depuis 1839 , ne le firent pas et se limitèrent à récupérer des vidanges pour réaliser des mélanges de type " noir animalisé ${ }^{121}$ ". Le rapport de l'inspecteur d'agriculture, Jules Vidal, en 1863, relativise nettement l'impact des guanos artificiels dans la consommation d'engrais artificiels en LoireInférieure comparativement aux noirs résidus et aux noirs animalisés, appelés ici " noirs composés " : "En première ligne viennent les noirs de raffinerie et les noirs composés, en seconde ligne les guanos péruviens, en troisième la chaux, en quatrième les boues des villes, en cinquième les charrées, puis enfin les guanos artificiels ${ }^{122}$. " Finalement, cette production de guano artificiel resta en Loire-Inférieure une production annexe de celle de noir animal et ne se substitua pas au négoce du guano du Pérou, qui s'amplifia. En 1862, les importations en Loire-Inférieure de guano artificiel représentaient 500

$19^{e}$ siècle, une victoire agronomique, condition de toutes les autres, INRA Mensuel, $\mathrm{n}^{\circ} 58$, 1991, p. 37-42).

119. Arch. dép. de Loire-Atlantique, 5M 381. Dossier Mongin (1858-1859), courrier de Mongin au préfet de Nantes le 11 octobre 1858.

120. Arch. dép. de Loire-Atlantique, 21U 60. Audience du tribunal de commerce de Nantes du 28 septembre 1861 entre Mongin et Burdelot; 21U 62, sauf-conduit du tribunal de commerce de Nantes du 11 septembre 1862; 21 U 739, jugement de faillite du 6 septembre 1862. Mongin, associé au négociant J. Burdelot, fit faillite en 1862 après dissolution de la société en 1861.

121. Arch. dép. de Loire-Atlantique, 21U 64. Audience du tribunal de commerce de Nantes du $1^{\text {er }}$ juillet 1863 entre Pilon père, Perthuy \& Cie et Bruneau. Les "noirs animalisés " coexistèrent avec le guano artificiel. Dans le département de l'Ille-et-Vilaine, nous ne trouvons pas de fabricants de guano artificiel dans les années 1845-1865, mais des fabricants d'" engrais animalisés " à côté d'ateliers d'équarrissage (Arch. dép. d'Ille-et-Vilaine, 5M 228-236). Á la campagne, dans les années 1855-1860, en périphérie de l'agglomération nantaise, les équarrisseurs installèrent aussi des ateliers de fabrication d'engrais à partir de chairs animales (Jumentier à Saint-Aignan, Miozé à Saint-Étienne-de-Montluc) (Arch. dép. de Loire-Atlantique, 7M 10, rapport au préfet de l'inspecteur d'agriculture, Jules Vidal, Nantes le 31 mars 1863; Arch. dép. de Loire-Atlantique, 5M 54).

122. Arch. dép. de Loire-Atlantique, 7M 10, Rapport au préfet de l'inspecteur d'agriculture, Jules Vidal, Nantes, le 31 mars 1863. " Il est utilisé dans le département, $125000 \mathrm{hl}$ en noirs de toutes sortes purs et composés, $1500000 \mathrm{~kg}$ de guanos, 20000 barriques de chaux, $8000 \mathrm{~m}^{3}$ de boues de ville, $12000 \mathrm{hl}$ de charrées et $15000 \mathrm{~kg}$ de guanos artificiels divers." 
tonnes ( $2 \%$ des importations totales) en provenance d'Angleterre face aux importations de noir animal en diminution de 17705 tonnes (46\%), celle de guano de 1500 tonnes (6,5 \%). Quant aux exportations de guano artificiel et engrais divers, elles s'élevaient à 500 tonnes $^{123}$.

Un tournant est sensible au milieu des années 1860 avec la manifestation de propos remettant en cause l'usage du terme guano. Le consignataire du gouvernement du Pérou à Paris y voyait « de grands abus avec le nom de guano " et estimait que " cette dénomination ne pût être appliquée qu'aux engrais naturels et non aux engrais artificiels ${ }^{124}$ ". De même, Jean Girardin décria leur côté trompeur dès 1864 et avec plus de virulence en 1876 : " Beaucoup de marchands ont eu la mauvaise idée de faire du mot guano un terme générique, un synonyme d'engrais; de là les dénominations vicieuses qui ont cours aujourd'hui [...] Il est bien évident que les auteurs ou vendeurs de ces compositions n'ont adopté cette fausse nomenclature que pour donner une haute idée de leurs mélanges et en faciliter plus aisément l'écoulement, parce qu'ils savent que les cultivateurs connaissent très bien aujourd'hui la puissante action des véritables guanos naturels ${ }^{125}$. " Cette contestation reflétait le début d'une désaffection pour ce produit ${ }^{126}$, probablement liée à la fin de l'engouement pour le guano naturel, dont les ressources commençaient à se tarir, et à l'arrivée d'autres produits azotés de récupération comme les eaux-vannes, le sulfate d'ammoniaque. Les changements de tendances se reflétèrent dans l'évolution des désignations des engrais d'Édouard Derrien, qui passèrent d'une dénomination initiale de " sels calcaires animalisés ${ }^{127}$ " apparenté au noir factice dans les années 1840, aux guanos artificiels dans les années 1850 et enfin aux « engrais artificiels Derrien ", dénomination assez neutre, dans les années 1860.

Dès les années 1870, le marché des engrais artificiels organiques déclina. Le guano artificiel « dont la dénomination [...] [prêtait] à l'équivoque [tendit] de plus en plus à disparaître ${ }^{128}$ " selon Bobierre. Il en fut de même pour les noirs résidus de raffinerie en raison de l'abandon du recours au noir animal pour la clarification du sucre brut après la mise au point de la filtration mécanique sur tissus ${ }^{129}$. Par ailleurs, alors qu'apparaissaient les " phospho-

123. BoBIERRE, Adolphe, " Le commerce des engrais dans la Loire-Inférieure ", Journal d'agriculture pratique, tome 2, 07 (année 26), 1862. Arch. dép. de Loire-Atlantique, 7M 10; rapport au préfet de l'inspecteur d'agriculture, Jules Vidal, Nantes le 31 mars 1863.

124. Dumas, Jean-Baptiste, Enquête sur les engrais, Paris, J. Rothschild éditeur, 1866, p. 89.

125. GIRARDIN, Jean, Des fumiers..., op. cit., 1876 ( $7^{\mathrm{e}}$ édition), p. 57-58.

126. Nous trouvons néanmoins encore en janvier 1904, Arthur Follen, à Dol-de-Bretagne en Ille-et-Vilaine, pour demander la création d'une fabrique de guano animal constitué par réduction des viandes, os et tous résidus et déchets au moyens d'acides (Arch. dép. d'Ille-et-Vilaine, 5M 233. Dossier Baguer-Morvan).

127. Cette mise en valeur du calcaire pourrait être liée au rôle important joué par le marnage dans la fertilisation des terres (DuBY, Georges et WALLON, Armand [dir], Histoire de la France rurale, 3. De 1789 à 1914, Paris, Éditions du Seuil, 1976, p. 111-114).

128. BOBIERRE, Adolphe, "Les engrais en Loire-Inférieure ", Journal d'agriculture pratique, tome 2, 07 (année 37), 1873.

129. Barles, Sabine, L'invention des déchets..., op. cit., p. 140-141. 
guano ", nouvelle sorte d'engrais composés, des critiques sur la confection des engrais composés par les industriels se firent jour ${ }^{130}$. L'industrie des engrais commençait à se désintéresser de matières premières urbaines et à mobiliser d'autres ressources comme le phosphate minéral, le nitrate de soude du Chili et la potasse allemande ayant un potentiel productif plus élevé $^{131}$. La grande industrie chimique amorça son emprise sur l'industrie des engrais. Dans le port de Nantes, la fabrique de noir animal et d'engrais d'Édouard Derrien fit faillite en $1878^{132}$. La société Toché fils reprit en 1880 la fabrique de noir animal de Benjamin Leroux pour y implanter une installation de fabrication d'acide sulfurique pour produire du superphosphate de chaux $^{133}$.

Le guano artificiel se situait à la croisée des enjeux d'autonomie en ressources, de réutilisation des rebuts industriels et urbains et de la qualité de produit. Il émergea en effet dans un contexte plus affirmé d'une crainte de l'épuisement des terres, d'une crainte de pénurie de fertilisant et d'une volonté d'autosuffisance face au commerce international des engrais. Il fut l'héritier d'engrais artificiels composés, comme le noir factice, apparu avec le commerce du noir résidu de raffinerie.

Des industriels, comme Édouard Derrien, dans l'agglomération nantaise, furent à l'origine de la reconnaissance de cette nouvelle filière des engrais artificiels manufacturés. Par un travail sur la qualité de leur produit, ils surent dépasser la suspicion de falsification en s'appropriant la normalisation de la définition des engrais et l'analyse chimique. Derrien s'appuya, en effet, sur les avancées de la réglementation du commerce des engrais en Loire-Inférieure pour faire reconnaître la qualité de son guano artificiel dans ses capacités fertilisantes. Il eut recours à l'appui de la communauté savante pour l'assister dans cette reconnaissance et conquérir ce nouveau marché au niveau national et international afin de développer son entreprise et cette nouvelle filière des engrais.

130. MüNTZ, Achille et GIRARD, Aimé, Les engrais, Tome III, Paris, Librairie de Firmin-Didot et $C^{\mathrm{ie}}, 1891$, p. 404-405 : " Les formules des engrais destinés aux diverses cultures sont établies, la plupart du temps, d'une manière tout à fait arbitraire par les fabricants; ceux d'entre eux qui sont plus instruits et plus soucieux de l'intérêt de leurs clients, tiennent compte de la composition des récoltes auxquelles on les destine, et de la proportion des éléments fertilisants nécessaires à la végétation. Admettons même que ces formules aient été établies sur des bases scientifiques, par des industriels au courant des principes de la physiologie végétale et de la chimie agricole; nous ne les critiquerons pas moins. Il ne faut pas, en effet, considérer seulement les besoins de la récolte, mais encore et surtout la richesse du sol, qui varie à l'infini et que les formules d'engrais ne peuvent pas prévoir. " 131. BARLEs, Sabine, L'invention des déchets..., op. cit., p. 135.

132. Arch. dép. de Loire-Atlantique, 21U 94. Audience du tribunal de commerce de Nantes du 30 novembre 1878 entre Péan et autres et syndic Derrien; 21U 95, Audience du tribunal de commerce de Nantes du 11 janvier 1879, maintien de syndic et liquidation Derrien, homologation de concordat Derrien; $21 \mathrm{U}$ 451, Publication des faillites, 16 juillet 1878. Faillite E. Derrien. Son actif s'élevait alors à 335000 francs.

133. Arch. dép. de Loire-Atlantique, 5M 383. Dossier Toché (1880). 
Le guano artificiel survint dans une période transitoire du développement de l'industrie des engrais dans l'estuaire de la Loire en marche vers sa maturité et sa reconnaissance. Il fut le résultat de la professionnalisation du fabricant d'engrais qui se démarqua de plus en plus du négociant spéculateur en appliquant une démarche rationnelle et expérimentale. Ce furent des fabricants de noir animal qui exploitèrent leur savoir-faire technique et chimique pour la fabrication d'engrais. La distinction négociant/industriel resta néanmoins encore floue. Bien qu'issue d'industriels en désaccord avec les nouvelles " doctrines des engrais chimiques", cette étape de l'industrie des engrais du milieu du XIX ${ }^{\mathrm{e}}$ siècle ouvrit la voie à une industrie des engrais de plus en plus transformatrice des matières et de plus en plus proche de la chimie, telle qu'elle se développera à la fin du siècle avec l'industrie chimique minérale des superphosphates. 


\section{RÉSUMÉ}

Cet article contribue à l'histoire des prémisses de l'industrie des engrais. C'est l'époque des débuts de l'agriculture intensive en France, une époque de transition où le fumier se révèle insuffisant face à un besoin croissant de terres à cultiver pour une population en forte hausse. C'est alors que se constitue le marché des engrais et s'élabore des réglementations de ce marché. L'engrais devient à la fois un enjeu du commerce international à la main du négoce et le produit d'une nouvelle industrie à la main d'industriels de plus en plus spécialisés dans sa fabrication.

Dans ce contexte d'accroissement de la demande de fertilisants, les industriels de l'estuaire de la Loire en proposent de nouveaux, des engrais composés à partir de résidus industriels et urbains locaux à bas prix. Ces produits se positionnent comme substituts de produits de ce commerce des " engrais artificiels " tels que les " noirs résidus de raffinerie " et les guanos du Pérou.

À travers une étude de cas, celle du fabricant d'engrais nantais, Edouard Derrien, nous nous proposons de mettre en lumière la stratégie relationnelle, industrielle et commerciale, mise en ouvre par ces fabricants du milieu du $\mathrm{XIX}^{\mathrm{e}}$ siècle en marche vers la professionnalisation. Nous suivrons leurs initiatives pour d'une part, amener à la maturité, avec des produits de qualités et innovants, cette industrie des " engrais artificiels " et pour, d'autre part, légitimer le métier de fabricant d'engrais confronté à la méfiance causée par la falsification des produits.

\section{ABSTRACT}

This paper contributes to the history of the premises of the fertiliser industry. It was the beginning of intensive agriculture in France, a time of transition where manure proved to be insufficient to cope with an increasing demand for farmland for a soaring population. The fertiliser market developed from this demand, as did industry regulations. Fertiliser became both an issue for international traders and a new product for industrialists that increasingly specialised in its manufacture.

In this context of increasing demand for fertilisers, industrialists of the Loire estuary proposed at low prices new fertiliser compounds manufactured from industrial and urban local residues. These products were offered as substitutes for "artificial fertilisers" such as "black refinery residues" and Peruvian guano.

Through a case study of the Nantes fertiliser manufacturer, Edouard Derrien, we intend to highlight the industrial and commercial relational strategy implemented by these mid-nineteenth century manufacturers as they became increasingly professionalised. We shall follow their initiatives as, on the one hand, they bring to maturity with innovative quality products this industry of "artificial fertilisers" and, on the other, legitimise the profession of fertiliser manufacturer despite the distrust caused by adulterated products. 